\title{
Asset Price Dynamics in a Financial Market with Fundamentalists and Chartists
}

\author{
CARL CHIARELLA $^{\mathrm{a}, *}$, ROBERTO DIECI $^{\mathrm{b}, \dagger}$ and LAURA GARDINI ${ }^{\mathrm{c}, \dagger}$ \\ ${ }^{a}$ School of Finance and Economics, University of Technology, Sydney; \\ ${ }^{\mathrm{b}}$ Facoltà di Economia, Università degli Studi di Parma; \\ ${ }^{\mathrm{c}}$ Facoltà di Economia, Università degli Studi di Parma and Università di Urbino
}

(Received 10 July 2000)

\begin{abstract}
In this paper we consider a model of the dynamics of speculative markets involving the interaction of fundamentalists and chartists. The dynamics of the model are driven by a two-dimensional map that in the space of the parameters displays regions of invertibility and noninvertibility. The paper focuses on a study of local and global bifurcations which drastically change the qualitative structure of the basins of attraction of several, often coexistent, attracting sets. We make use of the theory of critical curves associated with noninvertible maps, as well as of homoclinic bifurcations and homoclinic orbits of saddles in regimes of invertibility.
\end{abstract}

Keywords: Interacting agents; Noninvertible maps; Basins of attraction; Global bifurcations

\section{INTRODUCTION}

In this paper we shall develop, and investigate the dynamic behaviour of a discrete time model of asset price dynamics, which includes the one presented in [9] (and analyzed also in [15]) and can be reduced to the same two-dimensional dynamical system partially studied in [11]. We assume that the share market consists of two types of traders: fundamentalists, who are forming rational expectations on the fundamental value of the asset, and chartists, a group which bases its trading decisions on an analysis of past price trends. The chartist demand is assumed to be an $S$ shaped function of the difference between the chartists' estimate of the price trend (obtained through an adaptive expectations scheme on past price changes) and the return on some alternative asset. The model reduces to a two-dimensional nonlinear map. We study the influence on the asset price dynamics of the main parameters, such as the fundamentalists' strength of adjustment to the difference between the rationally expected fundamental value of the asset and the current price, and

\footnotetext{
*e-mail: carl.chiarella@uts.edu.au

${ }^{\dagger}$ Corresponding author. e-mail: roberto.dieci@unipr.it

‡e-mail: gardini@econ.uniurb.it
} 
the speed with which chartists adjust their estimate of the trend to past price changes.

The stability analysis of the unique equilibrium point of the map together with the regions of invertibility or non invertibility give a good idea of the dynamical behaviour locally, in a neighbourhood of the equilibrium point. However, it is only through the global analysis of the basin of attraction that we are able to understand how the stable equilibrium may sometimes be considered as "practically unstable", in the sense that its basin of attraction is very small and displays a fractal structure, or the equilibrium point is in competition with other attractors. The aim of the paper is therefore mainly to show how situations may occur that seem counter-intuitive: regions of the parameters space where the equilibrium point is locally stable are often characterized by practical instability, because its basin is very small, often with a complex structure, and/or in competition with a coexisting attractor (either diverging trajectories or another bounded attractor). It is important to stress that this kind of analysis of the basin structure can be performed only through numerical simulations: this is in fact the only way to detect global bifurcations occurring as a consequence of contacts between curves (for example critical curves and basin boundaries or stable and unstable sets) whose analytical expression generally cannot be determined.

It was a very pleasant surprise for us to discover that the economic model described in Section 2 leads to the same two-dimensional map governing the dynamical behaviour of the model proposed in [11], and there partially studied. This map also generalizes the one analysed in [9]. Thus we shall briefly recall some results on the local analysis of the model, referring to [11] for comments and explanations concerning some types of global behaviour. In this paper we are particularly interested in the study of dynamic behaviour which turns out to be different to that analyzed in the previous paper [11]. While in [11] attention was focussed on the homoclinic bifurcation of a repelling focus known as a "snap-back-repeller", here we shall consider the homoclinic bifurcations of saddle cycles, both in the case of invertible and noninvertible maps. Moreover in [11] it was observed, without however any explanation, that a new kind of mechanism leading to fractalization of the basins is associated with noninvertible maps. In this paper we shall explain in detail how this "route" works, in particular how it is associated with contact bifurcations involving the critical curves of the map and other invariant sets. However we shall also see a "route" similar to the one occurring in invertible maps.

The paper is organized as follows. Section 2 derives our model of fundamentalists and chartists and recalls some general properties of the twodimensional map driving the dynamics. Section 3 gives the detailed analysis of the role of the noninvertibility of the map in determining a sequence of global bifurcations which profoundly modify the basin of attraction of the fixed point and lead to fractalization and to a situation of "practical instability". Section 4 deals with a global bifurcation causing the disappearance of a closed invariant curve born at a Neimark-Hopf bifurcation. It is due to a homoclinic connection, not related to chaotic dynamics, differently from the cases presented in Section 3. Section 5 presents a different route to fractalization of the basins, which is the standard one in invertible maps, associated with the transverse homoclinic orbits of saddle cycles. While in Section 6 we shall see how the transition from a "wide basin" of the locally stable fixed point to a "small basin" can occur via the coexistence with several kinds of attracting sets (regular cycles or chaotic sets) which dominate in the Lebesgue measure, and undergo an interesting sequence of "forward" and "backward" global bifurcations. Section 7 offers some conclusions and suggestions for further research.

\section{THE MODEL}

Let us denote by $P_{t}$ the logarithm of the asset price at time $t$. Excess demand for the asset at time $t\left(D_{t}\right)$ 
is composed of fundamentalist demand $\left(D_{t}^{0}\right)$ and chartist demand $\left(d_{t}\right)$, i.e.:

$$
D_{t}=D_{t}^{0}+d_{t} .
$$

Fundamentalist demand is given by:

$$
D_{t}^{0}=a\left(W_{t}-P_{t}\right),
$$

where $W_{t}$ is the logarithm of the price that clears fundamental demand at time $t$ (i.e., the fundamental value at time $t)$ and $a(a>0)$ is the fundamentalists' strength of adjustment to the difference between the rationally expected fundamental value of the asset and the current price. Chartist demand is assumed to be a nonlinear function of the anticipated return differential $\left(\psi_{t, t+1}-g_{t}\right)$, where $\psi_{t, t+1}$ is the chartists' expectation at time $t$ of the price change between $t$ and $t+1$, i.e.,

$$
\psi_{t, t+1}=E_{t}\left[P_{t+1}-P_{t}\right]=E_{t}\left[P_{t+1}\right]-P_{t}
$$

and $g_{t}$ is the return on the alternative asset (bonds) over the same period. In particular we write:

$$
d_{t}=h\left(\psi_{t, t+1}-g_{t}\right)
$$

where, following [9], the function $h(\cdot)$ has the following properties:

- $h^{\prime}(x)>0$ for all $x$

- $h(0)=0$

- there exists an $x^{*}$ such that $h^{\prime \prime}(x)<0(>0)$ for all $x>x^{*}\left(<x^{*}\right)$;

- $\lim _{x \rightarrow \mp \infty} h^{\prime}(x)=0$.

The economic scenario behind the demand function $h$ is one in which chartists allocate their wealth (fixed in the short term) between the risky asset and the alternative safe asset depending on the expected return differential $\left(\psi_{t, t+1}-g_{t}\right)$. As this differential assumes extreme values the demand moves towards "all in the risky asset" or "all in the alternative asset".

A possible choice for the function $h(\cdot)$ is

$$
h(x)=\alpha \arctan x
$$

and this is the one used in our examples and simulations. However, it is important to remark that the qualitative analysis performed in the following sections (as also the qualitative dynamics) are not affected by a change of function, because these mainly depend on the properties of $h(\cdot)$ given above.

Chartists are assumed to form their expectation of the price change adaptively, i.e.,

$$
\psi_{t, t+1}=\psi_{t-1, t}+c\left[\boldsymbol{P}_{t}-P_{t-1}-\psi_{t-1, t}\right],
$$

where $c(c>0)$ is the speed with which chartists adjust their estimate of the trend to past price changes. ${ }^{1}$

Total excess demand for the asset at time $t$ is given by:

$$
D_{t}=a\left(W_{t}-P_{t}\right)+h\left(\psi_{t, t+1}-g_{t}\right),
$$

while the asset price is assumed to adjust with some delay to excess demand, ${ }^{2}$ i.e.,

$$
P_{t+1}=P_{t}+\beta_{p}\left[a\left(W_{t}-P_{t}\right)+h\left(\psi_{t, t+1}-g_{t}\right)\right],
$$

where $\beta_{p}$ (a positive constant) is the speed of adjustment of the asset price to excess demand.

Given the current price and the previous one $\left(P_{t}\right.$ and $\left.P_{t-1}\right)$, and the most recent expectation of the price change $\left(\psi_{t-1, t}\right)$, the system (1)-(2), i.e.,

$$
\left\{\begin{array}{l}
\psi_{t, t+1}=\psi_{t-1, t}+c\left[\left(P_{t}-P_{t-1}\right)-\psi_{t-1, t}\right] \\
P_{t+1}=P_{t}+\beta_{p}\left[a\left(W_{t}-P_{t}\right)+h\left(\psi_{t, t+1}-g_{t}\right)\right]
\end{array}\right.
$$

models the formation of the new expectation, $\psi_{t, t+1}$, and then of the new price, $P_{t+1}$. We note

\footnotetext{
${ }^{1}$ The further restriction $c \leq 1$ (which requires that chartists cannot revise their estimate of $\psi_{t, t+1}$ more frequently than they receive information about price changes) is particularly relevant from an economic point of view (see [11]).

${ }^{2}$ The economic scenario behind Eq. (2) is that of a market maker announcing prices in one period as a function of excess demand in the previous period. See [11] for more details.
} 
that by assuming $W_{t}$ and $g_{t}$ constant, i.e., $W_{t}=W$, $g_{t}=g$, we are dealing with a system which seems, at a first glance, a three dimensional one, while it can in fact be reduced to a two-dimensional system. We can rewrite (3) as:

$$
\left\{\begin{array}{l}
P_{t}=Z_{t-1} \\
\psi_{t, t+1}=(1-c) \psi_{t-1, t}+c\left(Z_{t-1}-P_{t-1}\right) \\
Z_{t}=Z_{t-1}+\beta_{p}\left[a\left(W-Z_{t-1}\right)+h\left(\psi_{t, t+1}-g\right)\right]
\end{array},\right.
$$

or:

$$
\left\{\begin{array}{l}
P^{\prime}=Z \\
\psi^{\prime}=(1-c) \psi+c(Z-P) \\
Z^{\prime}=Z+\beta_{p}\left[a(W-Z)+h\left(\psi^{\prime}-g\right)\right]
\end{array},\right.
$$

where the symbol ' denotes the unit time advancement operator, that is, if $P, \psi$ and $Z$ are the state variables at time $(t-1)$, then $P^{\prime}, \psi^{\prime}$ and $Z^{\prime}$ are the state variables at time $t$. The three dimensional system (4) can be reduced by observing that the $Z$ variable is simply an auxiliary, or phantom, variable. In fact, by using the third equation of (4) and the first one, we can explicitely write the $Z$ variable as a function of $P$ and $\psi$, as follows:

$$
Z=P+\beta_{p}[a(W-P)+h(\psi-g)]
$$

and substituting this expression into the first two equations of (4), we get:

$$
\left\{\begin{array}{l}
P^{\prime}=P+\beta_{p}[a(W-P)+h(\psi-g)] \\
\psi^{\prime}=(1-c) \psi+c \beta_{p}[a(W-P)+h(\psi-g)]
\end{array},\right.
$$

while it is easy to check that the third equation in (4) is redundant since it reduces again to the first equation in (5).

Thus the original system (3) has been reduced to the two-dimensional iterative process in (5), which at each time models the formation of the new price and then of the chartists' expectation of the price change over the next period, on the basis of the actual price and of the most recently calculated expectation. We also note that with the assumption $W_{t}$ and $g_{t}$ constant, system (5) is immediately obtained from (3) by writing the iterative process as follows:

$$
\begin{aligned}
P_{t}= & P_{t-1}+\beta_{p}\left[a\left(W-P_{t-1}\right)+h\left(\psi_{t-1, t}-g\right)\right] \\
\psi_{t, t+1}= & (1-c) \psi_{t-1, t}+c\left(P_{t}-P_{t-1}\right) \\
= & (1-c) \psi_{t-1, t} \\
& +c \beta_{p}\left[a\left(W-P_{t-1}\right)+h\left(\psi_{t-1, t}-g\right)\right]
\end{aligned}
$$

This particular two-dimensional system (5) can also be directly deduced following a different approach, based on the concept of market maker, as shown in [11]. Finally, it is worth noting that the model includes, as a particular case, the one developed in [9] (and analyzed also in [15]), starting from different assumptions about the chartists' behaviour. This fact enhances the value of the model and increases the importance of its analysis.

The study of the dynamical properties of the system (5) allows us to explore the long-run behaviour of price and chartists' expectations, starting from a given initial condition. Besides the situations already described in [11], new ones will be shown in Sections 3-6 of this paper. But let us first recall some basic properties of the system (5).

\subsection{Some General Properties}

We shall now recall from [11] some simple properties of the two dimensional map "driving" the discrete dynamical system (5). That is, the local stability analysis of the fixed point and the discussion of the regimes of invertibility and non invertibility of the map.

As it is easy to see the system (5) has a unique equilibrium point, given by the solution of the system:

$$
\left\{\begin{array}{l}
\beta_{p}[a(W-P)+h(\psi-g)]=0 \\
-c \psi+c \beta_{p}[a(W-P)+h(\psi-g)]=0
\end{array}\right.
$$


that is ${ }^{3}$

$$
\left\{\begin{array}{l}
P=W+1 / a h(-g) \\
\psi=0
\end{array} .\right.
$$

It is useful to rewrite the system (5) in an equivalent form, by introducing the price deviation $p=P-(W+1 / a h(-g))$, so that the time evolution of the dynamic variables $p$ and $\psi$ is driven by the two-dimensional map $T:(\psi, p) \rightarrow\left(\psi^{\prime}, p^{\prime}\right)$ given by:

$$
T:\left\{\begin{array}{l}
\psi^{\prime}=(1-c) \psi-c \beta_{p}[a p-k(\psi)] \\
p^{\prime}=p-\beta_{p}[a p-k(\psi)]
\end{array}\right.
$$

where:

$$
k(\psi)=h(\psi-g)-h(-g),
$$

whose unique fixed point is the origin $O=(0,0)$.

\subsection{Local Stability Analysis of the Fixed Point}

It is well known that the local stability of a fixed point depends on the eigenvalues of the Jacobian matrix evaluated at the fixed point. If we denote by $k^{\prime}(\psi)$ the first derivative of the function $k(\psi)$, the Jacobian matrix of $T$ is given by:

$$
D T(\psi, p)=\left[\begin{array}{cc}
1-c+c \beta_{p} k^{\prime}(\psi) & -a c \beta_{p} \\
\beta_{p} k^{\prime}(\psi) & 1-a \beta_{p}
\end{array}\right]
$$

which is independent of $p$. A sufficient condition for the local stability of the fixed point $O=(0,0)$ is that the eigenvalues of:

$$
D T(0,0)=\left[\begin{array}{cc}
1-c+c \beta_{p} k^{\prime}(0) & -a c \beta_{p} \\
\beta_{p} k^{\prime}(0) & 1-a \beta_{p}
\end{array}\right]
$$

are inside the unit circle in the complex plane, i.e., that the following conditions are satisfied (see, for instance, [21], p. 159, or [17])

$$
\left\{\begin{array}{l}
\mathcal{P}(1)=1-\operatorname{Tr}+\operatorname{Det}>0 \\
\mathcal{P}(-1)=1+\operatorname{Tr}+\operatorname{Det}>0 \\
\mathcal{P}(0)=\operatorname{Det}<1
\end{array}\right.
$$

where:

$$
\operatorname{Tr}=2-a \beta_{p}-c+c \beta_{p} k^{\prime}(0)
$$

and

$$
\operatorname{Det}=\left(1-a \beta_{p}\right)(1-c)+c \beta_{p} k^{\prime}(0)
$$

are, respectively, the trace and the determinant of (9), and $\mathcal{P}(\lambda)=\lambda^{2}-\operatorname{Tr} \lambda+$ Det is its characteristic polynomial. The first condition in (10) is equivalent to:

$$
a \beta_{p} c>0
$$

always being true. ${ }^{4}$

As far as the second and third conditions in (10) are concerned, elementary computations show that they can be rewritten, respectively, as:

$$
\begin{gathered}
a \beta_{p}(2-c)<2(2-c)+2 c \beta_{p} k^{\prime}(0) ; \\
a \beta_{p}(1-c)>c\left[\beta_{p} k^{\prime}(0)-1\right] .
\end{gathered}
$$

Figure 1 represents the region (indicated in green) of local stability of the origin in the parameter plane $(c, a), c>0, a>0$. The shape of this region is affected by the remaining parameters, strength of chartists' demand at the steady state $\left(k^{\prime}(0)\right)$ and the speed of adjustment of market price $\left(\beta_{p}\right)$. From a qualitative point of view, we can distinguish two cases: case A (represented in Fig. 1a), with $\beta_{p} k^{\prime}(0) \leq 1$, and case $\mathrm{B}$ (Fig. $1 \mathrm{~b}$ ), with $\beta_{p} k^{\prime}(0)>1$. From Eqs. (11) and (12) it follows that starting from inside the stability region in the parameter

\footnotetext{
${ }^{3}$ The equilibrium value of $P$ seems counter-intuitive in that it is not equal to $W$, the rationally expected fundamental value. This is entirely due to the fact that our economic analysis is a partial one. A proper modelling would require us to also model the dynamics of the price of the alternative asset (see [12]). However since we ultimately work in terms of deviation from the equilibrium we feel that the partial analysis is justified, particularly as it allows to work with a two-dimensional, rather than three-dimensional, map.

${ }^{4}$ We recall that only positive values of the parameters make sense economically.
} 


\section{CASE A}

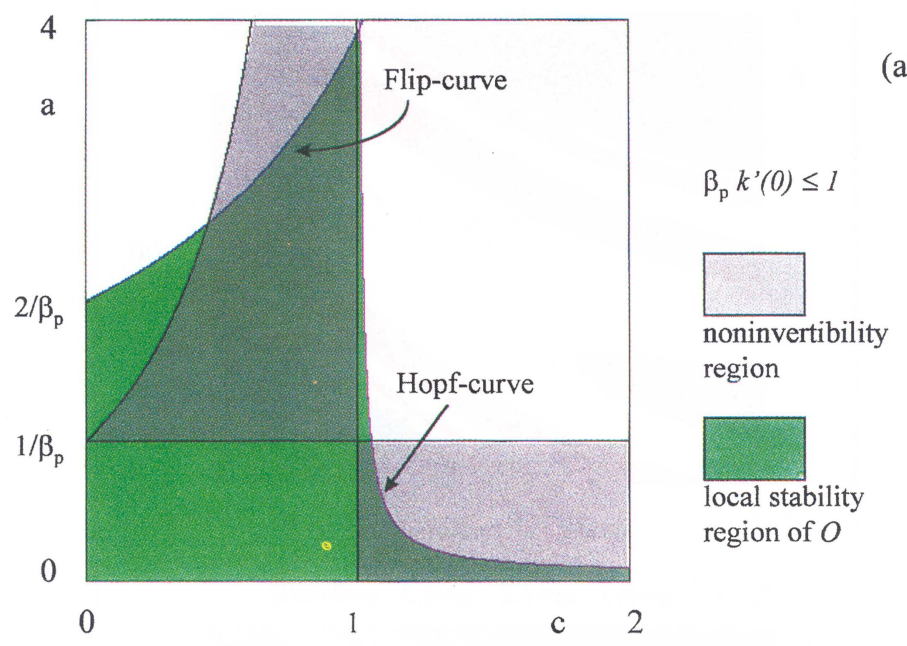

\section{CASE B}

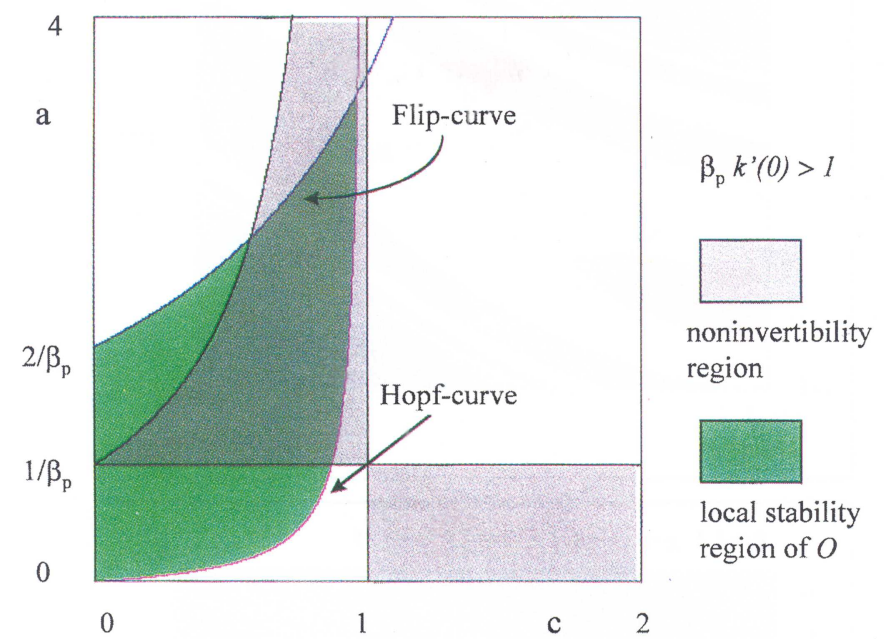

(b)

FIGURE 1 The green regions in (a) and (b) represent the stability regions of the equilibrium point $O$ in the plane of the parameters $c$ (speed of adjustment of chartists' expectations) and $a$ (strength of fundamentalists' demand), while the grey regions represent the domain of noninvertibility of the map $T$ in the same $(c, a)$ parameter plane. Figure (a) is obtained with values of the parameters $\beta_{p}$, $\alpha, g$ such that $\beta_{p} k^{\prime}(0)=\beta_{p} \alpha /\left(1+g^{2}\right) \leq 1$. In this case (case A) the domain of stability includes any value $c \in(0,1)$ for sufficiently low values of $a$ and the stability can be lost only via a Flip bifurcation as $a$ is increased. Figure (b) describes the opposite case (case B, $\beta_{p} k^{\prime}(0)>1$ ), where stability can be lost also via a Neimark-Hopf bifurcation. This figure also shows that for sufficiently low values of $a$ the map is invertible for any value $c \in(0,1)$ and comparing (a) and (b) we see that the stability region is wider in case A than in case B. (See Color Plate I.)

plane $(c, a)$, and increasing the chartists' adjustment parameter $c$ or the fundamentalists' parameter $a$, a loss of stability may occur either via a flip bifurcation, when crossing the curve

$$
a=\frac{2}{\beta_{p}}+\frac{2 c k^{\prime}(0)}{2-c}
$$

or via a Neimark-Hopf bifurcation, when crossing the curve

$$
a=\frac{c\left[\beta_{p} k^{\prime}(0)-1\right]}{\beta_{p}(1-c)}
$$

In particular, in case $A$ the Neimark-Hopf bifurcation cannot occur when $c$ ranges from 0 
to 1 (i.e., for values of the chartists' parameter which are meaningful from an economic point of view).

\subsection{Invertibility Conditions}

For particular values of the parameters, the map $T$ is a noninvertible map of the plane. This means that while starting from some initial values for chartists' expectations and asset price, say $\left(\psi_{0,1}\right.$, $p_{0}$ ), the iteration of (7) uniquely defines the trajectory $\left(\psi_{t, t+1}, p_{t}\right)=T^{t}\left(\psi_{0,1}, p_{0}\right), \quad t=1,2, \ldots$, the backward iteration of (7) may be not uniquely defined. In fact, a point $(\psi, p)$ of the plane may have several rank-1 preimages.

Let us assume:

$$
h(\cdot)=\alpha \arctan (\cdot) \quad(\alpha>0),
$$

so that

$$
k(\psi)=\alpha \arctan (\psi-g)-\alpha \arctan (-g) .
$$

In [11] conditions on the parameters, $\alpha, \beta, a, c$, are provided under which the map is noninvertible. Precisely, it is easy to show with elementary geometrical tools that, by defining:

$$
m=\left(a \beta_{p}-1\right) \frac{(1-c)}{c},
$$

the map has a unique inverse for $m \leq 0$ or $m \geq \alpha \beta_{p}$, while for $0<m<\alpha \beta_{p}$ the map is noninvertible. In particular, by defining:

$$
\begin{aligned}
& \psi_{1}=g-\sqrt{\frac{\alpha \beta_{p}}{m}-1}, \quad q_{1}=\beta_{p} k\left(\psi_{1}\right)-m \psi_{1} \\
& \psi_{2}=g+\sqrt{\frac{\alpha \beta_{p}}{m}-1}, \quad q_{2}=\beta_{p} k\left(\psi_{2}\right)-m \psi_{2}
\end{aligned}
$$

it is possible to show that the points $(\psi, p)$ of the phase plane for which

$$
q_{1}<\left(a \beta_{p} p-\frac{a \beta_{p}-1}{c} \psi\right)<q_{2},
$$

have three distinct rank-1 preimages, while the points satisfying

$$
\begin{aligned}
& \left(a \beta_{p} p-\frac{a \beta_{p}-1}{c} \psi\right)<q_{1} \text { or } \\
& \left(a \beta_{p} p-\frac{a \beta_{p}-1}{c} \psi\right)>q_{2}
\end{aligned}
$$

have a unique rank-1 preimage. Thus, following the notation used in [27], for $0<m<\alpha \beta_{p}$ this map is of the type $Z_{1}-Z_{3}-Z_{1}$, which means that the phase plane is subdivided into different regions $Z_{j}$ $(j=1,3)$ each point of which has $j$ distinct rank-1 preimages. Such regions are bounded by the socalled critical curves of rank-1 (see [21]), defined as the locus of points having at least two merging rank-1 preimages. Then by defining:

$$
q(\psi, p)=a \beta_{p} p-\frac{a \beta_{p}-1}{c} \psi,
$$

we can observe that for our map this set is defined as follows:

$$
L C=\left\{(\psi, p) \in \mathbf{R}^{2}: q(\psi, p)=q_{1} \cup q(\psi, p)=q_{2}\right\},
$$

where $q_{1}$ and $q_{2}$ are given in (16) and (17) respectively. The locus $L C$ is therefore made up of two straight lines, say $L C=L \cup L^{\prime}$, where $L$ and $L^{\prime}$ have the equations:

$$
\begin{aligned}
& L: \quad p=\frac{a \beta_{p}-1}{a \beta_{p} c} \psi+\frac{q_{1}}{a \beta_{p}} \\
& L^{\prime}: \quad p=\frac{a \beta_{p}-1}{a \beta_{p} c} \psi+\frac{q_{2}}{a \beta_{p}} .
\end{aligned}
$$

Each of the critical points $(\psi, p) \in L C$ has two merging rank-1 preimages, and the locus of such preimages, denoted by $L_{-1}$ (critical curve of rank-0), is made up of the two straight lines, say $L C_{-1}=L_{-1} \cup L_{-1}^{\prime}$, of equation:

$$
\begin{array}{ll}
L_{-1}: \quad \psi=g-\sqrt{\frac{\alpha \beta_{p}}{m}-1} & \\
L_{-1}^{\prime}: \quad \psi=g+\sqrt{\frac{\alpha \beta_{p}}{m}-1},
\end{array}
$$


The critical curve $L C_{-1}$ corresponds here to the locus of points $(\psi, p)$ of the phase plane in which the determinant of the Jacobian matrix $D T(\psi, p)$ vanishes.

Also the images of this set are called critical curves of higher rank. The curves:

$$
L C_{k}=T^{k}(L C)=T^{k+1}\left(L C_{-1}\right) \quad k=0,1,2, \ldots
$$

are called critical curves of rank- $(k+1)\left(L C_{0}=\right.$ $L C)$. In our example we always have two branches:

$$
L C_{k}=L_{k} \cup L_{k}^{\prime}=T^{k+1}\left(L_{-1}\right) \cup T^{k+1}\left(L_{-1}^{\prime}\right) .
$$

The important role of the technique of critical curves in describing several bifurcation mechanisms has already been pointed out in the economic literature (see for example $[4,5]$ and the recent monograph [31]). This technique will also play an important role in the following sections.

In Figure 1, together with the bifurcation curves in the parameter plane $(c, a)$, we have drawn also the region (indicated in grey) of non invertibility of the map $T$. In the $(c, a)$ plane the region in which the noninvertibility condition $0<m<\alpha \beta_{p}$ is fulfilled is given by:

$$
\left\{\begin{array} { l } 
{ 0 < c < 1 } \\
{ 1 / \beta _ { p } < a < 1 / \beta _ { p } + \alpha c / ( 1 - c ) }
\end{array} \cup \left\{\begin{array}{l}
c>1 \\
a<1 / \beta_{p}
\end{array} .\right.\right.
$$

\section{COMPLEX BASIN BOUNDARIES FOR THE NONINVERTIBLE MAP}

In this section we shall consider a few examples of "routes-to-complex-basins" related to situations included in case B, which is described in Section 2.2 and illustrated in Figure $1 \mathrm{~b}$. In that parameter situation in fact, fixing $a>1 / \beta_{p}$, the stability region of the fixed point $O$ in the parameter plane $(c, a)$ mainly belongs to the noninvertibility region for $T$, and as the parameter $c$ increases the Neimark-Hopf bifurcation curve is necessarily met. However, even if we are in the region in which the fixed point is locally stable, we may have a surprisingly small basin of attraction and/or of so complex a structure such that the local stability may appear as "practical instability" from a global point of view. We shall denote by $\mathcal{B}(O)$ the basin of attraction of the fixed point of $T$, when it is locally stable. That is, $\mathcal{B}(O)$ is the locus of points of the plane (states of the system) whose trajectory converges to the equilibrium. By $\mathcal{B}(\infty)$ we denote the points of the plane having divergent trajectories. The existence of such trajectories denotes an attracting set at infinity (on the so-called Poincaré Equator), so that we may also denote this set $\mathcal{B}(\infty)$ as the basin of the points at infinity (or basin of the Poincare Equator). As long as the fixed point is the only bounded attractor, we have that the sets $\mathcal{B}(O)$ and $\mathcal{B}(\infty)$ are a kind of complementary sets. To be precise, $\overline{\mathcal{B}(\infty)}=\mathcal{C}(\mathcal{B}(O))$, that is, the closure of $\mathcal{B}(\infty)$ is the complementary set of $\mathcal{B}(O)(\mathcal{C}(\cdot)$ denotes the complement and an overbar denotes the closure). So that the two sets $\overline{\mathcal{B}(\infty)}$ and $\mathcal{B}(O)$ cover the whole plane and have the same frontier (or boundary) $\partial \mathcal{B}(\infty)=\partial \mathcal{B}(O)$ given by $\overline{\mathcal{B}(O)} \cap \overline{\mathcal{B}(\infty)}{ }^{5}$ This is no longer true when two or more bounded attractors exist, as we shall see in Section 6 . When there are coexisting attractors the set $\mathcal{B}(\infty)$ always denotes the set of divergent trajectories, and its complementary set $\mathcal{C}(\mathcal{B}(\infty))$ the locus of points of the phase-plane having bounded limit sets (or $\omega$-limits). Then $\mathcal{C}(\overline{\mathcal{B}(\infty)})$ is shared between two or more attractors and includes two or more basins.

In the present section we shall see how the increasing complexity in the structure of the basins of attraction may be caused by global bifurcations (or contact bifurcations) involving the critical curves of the map. Let us fix the parameters $\beta_{p}=2.5, g=0.5, \alpha=2.3, a=1.8$, and increase $c$ from the value 0.49 . The fixed point $O$ is a stable focus, and it will undergo a Neimark-Hopf bifurcation at $c=0 . \overline{5}$.

\footnotetext{
${ }^{5}$ We recall that the frontier of a subset $A$ of the plane is given by the intersection between the closure of $A$ and the closure of its complementary set, $\partial A=\bar{A} \cap \overline{C A}$. Or, stated in a different way, a point $\mathbf{x}$ belongs to the frontier of $A$ if in any neighbourhood of $A$ there are points belonging to $A$ as well as points belonging to the complement of $A$.
} 
But what about the global dynamics? What is the nature of the basin of attraction of the stable fixed point? In general it is difficult to give an answer to such questions without the help of numerical simulations: such "guided" experiments on fixed examples are in fact the only way to detect the occurrence of global bifurcations related to contacts between curves (critical curves and basin boundaries) whose analytical expression not only cannot be found (this is the case, for example, of the critical curves of high rank) but also often does not exist (this case normally occurs for fractal basin boundaries).

We shall see that in the regime of noninvertibility of $T$ the critical curves will play an important role for the analysis of the basin of attraction of the stable fixed point: in fact, this basin will undergo global bifurcations, and the basin structure will be deeply modified, as a consequence of contacts with the critical curves. In Figure 2a we can see that the basin of the origin (the white region whose boundary includes the stable set of a cycle of saddle type) is a connected area, and the grey points denote the basin of infinity $\mathcal{B}(\infty)$. However, from the same figure we can see that there are arcs on the boundary $\partial \mathcal{B}(O)=\partial \mathcal{B}(\infty)$ which approach the critical curve $L^{\prime}$ (see the arrow in Fig. 2a). The strip between the two critical lines $L$ and $L^{\prime}$ is the region $Z_{3}$ of points having three distinct rank-1 preimages, while the regions outside are $Z_{1}$ and $Z_{1}^{\prime}$, whose points have a unique preimage. Each point of $Z_{3}$ has one preimage, say $T_{(1)}^{-1}$, in the strip between the vertical lines $L_{-1}$ and $L_{-1}^{\prime}$, a second preimage, say $T_{(2)}^{-1}$, on the right of $L_{-1}^{\prime}$, and a third one, say $T_{(3)}^{-1}$, on the left of $L_{-1}$. The points $\mathbf{x}$ belonging to $Z_{1}$ below (resp. $Z_{1}^{\prime}$ above) the strip $Z_{3}$ only have the preimage $T_{(2)}^{-1}(\mathbf{x})\left(\right.$ resp. $\left.T_{(3)}^{-1}(\mathbf{x})\right)$.

When $\partial \mathcal{B}(O)$ has a contact with $L^{\prime}$ we have a bifurcation in the structure of the basin $\mathcal{B}(O)$. Such a contact with $L C$ will cause the appearance of "islands" of $\mathcal{B}(\infty)$, or "holes" of $\mathcal{B}(O)$, made up of points having divergent trajectories also inside the old white area, thus rendering the basin $\mathcal{B}(O)$ a multiconnected region (i.e., a region with holes). This can be seen in Figure $2 b$, the frontier $\partial \mathcal{B}$ intersects $L^{\prime}$ and the portion of $\mathcal{B}(\infty)$ below $L^{\prime}$, denoted $H_{0}$ in Figure $2 \mathrm{~b}$ is a portion of $\mathcal{B}(\infty)$ which has crossed the boundary $L^{\prime}$ entering the

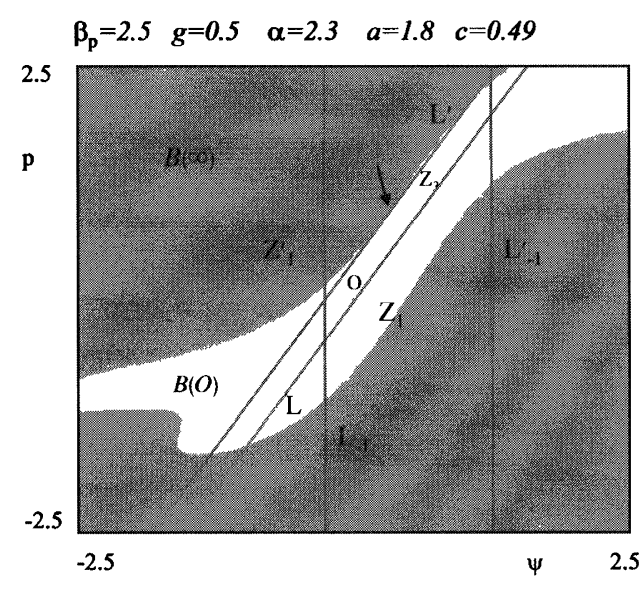

(a)

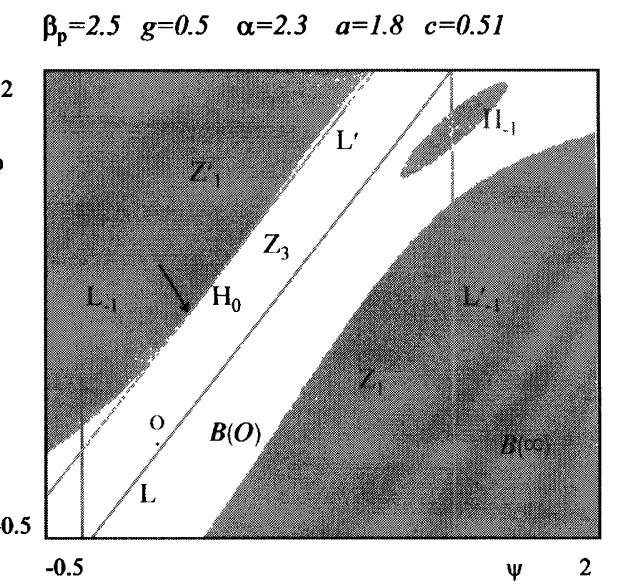

(b)

FIGURE 2 Basin bifurcations of the stable fixed point $O=(0,0)$, observed in the regime of noninvertibility of the map $T$, for $\beta_{p}=2.5, g=0.5, \alpha=2.3, a=1.8$ and by increasing $c$ from the value 0.49 . As explained in the text, the critical curves of the map allow one to explain the qualitative changes in the basin structure. At $c=0.49$ in (a), where the basin of the origin, the white region $\mathcal{B}(O)$, is a simply connected area. At $c=0.51$ in (b) and (c), where the grey region $\mathcal{B}(\infty)$ crosses the critical curve $L^{\prime}$, entering the region $Z_{3}$ and $\mathcal{B}(O)$ is a multiply connected area. At $c=0.533$ in (d), where a new crossing of the boundary $L$ causes the appearance of new holes. A different dynamic effect is associated with a similar crossing by the hole $S_{-1}$ : in (e), where $c=0.534$, it is shown that differently from the previous cases the hole $Q_{-1}$ generated by the new crossing is now in the region $Z_{3}$, and thus it has three distinct rank-1 preimages, each of which has one or three preimages, so that a drastic increase in the numbers of the holes is observed, leading to an increasing complexity in the structure of the basin $\mathcal{B}(O)$. This kind of bifurcation also occurs in a "backward" way, with the effect of decreasing the number of holes, as shown in (f), obtained for $c=0.55$. 

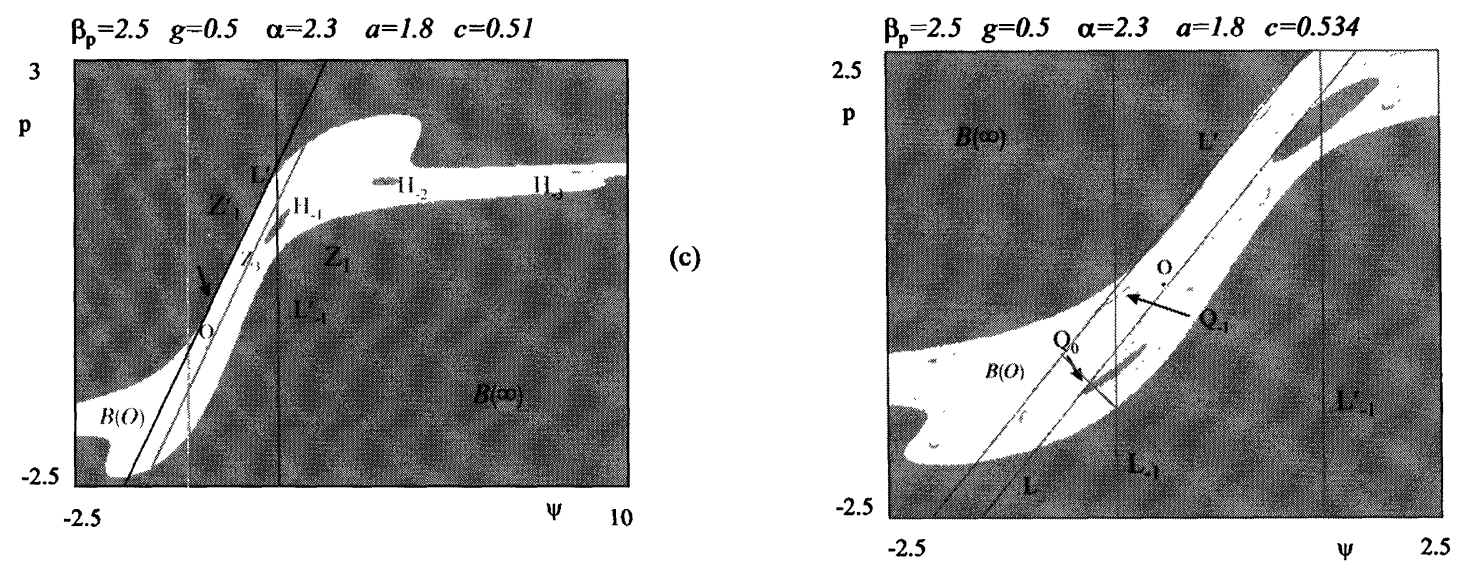

(e)

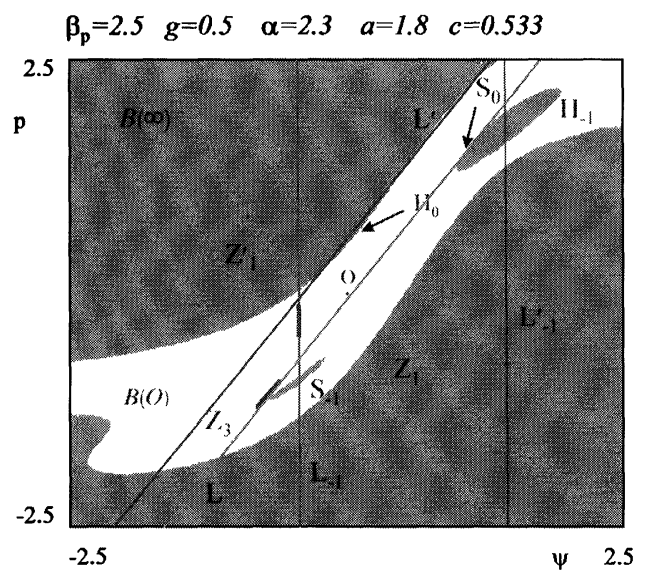

(d)

FIGURE 2 (Continued).

region $Z_{3}$ with three distinct preimages, and thus the small portion $H_{0}$ has three distinct rank-1 preimages, two of which are on opposite sides of the line $L_{-1}^{\prime}$, one on the right and one on the left, connected through a segment of $L_{-1}^{\prime}$, constituting the set denoted as $H_{-1}$ in Figure 2b, $H_{-1}=T_{(1)}^{-1}\left(H_{0}\right) \cup T_{(2)}^{-1}\left(H_{0}\right)$. The third preimage $T_{(3)}^{-1}\left(H_{0}\right)$ is in the grey region on the left of $L_{-1}$. The "island" or "hole" $H_{-1}$ is a new portion which did not exist before the contact bifurcation between $\partial \mathcal{B}$ and $L C$, and is made up of points having divergent trajectories, and the same holds for an infinite sequence of "islands" obtained by taking all the preimages of any rank of $H_{-1}$, only a few of which are visible in Figure 2c. Since $H_{-1}$ is completely included in $Z_{1}$, below the strip $Z_{3}$, its

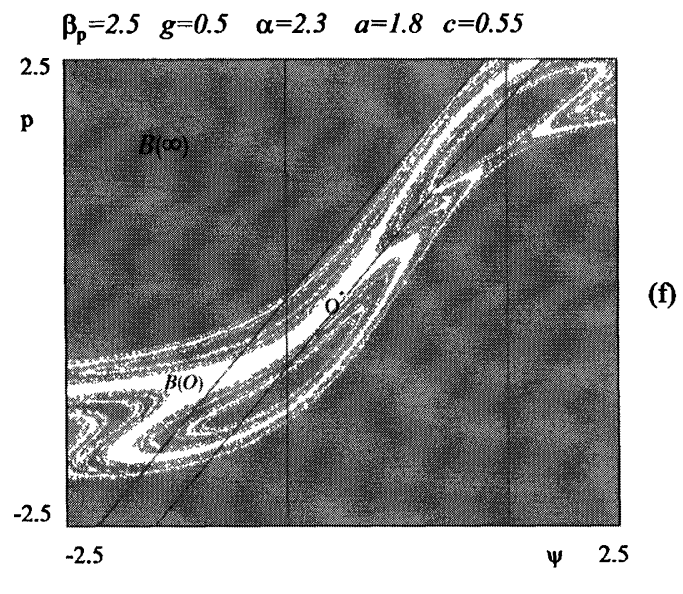

FIGURE 2 (Continued).

preimage is $T_{(2)}^{-1}\left(H_{-1}\right)=H_{-2}$ again in $Z_{1}$, and so on, $T_{(2)}^{-1}\left(H_{-2}\right)=H_{-3}, \ldots$, an infinite sequence exists, given by $T_{(2)}^{-k}\left(H_{-1}\right)$, for $k=1,2, \ldots$, which are "islands" appearing further and further from the origin and approaching the Poincare Equator.

This bifurcation structure has been discussed for the first time in [25] (see also [27, 1,26]). It is clear that other contacts of $\partial \mathcal{B}(\infty)$ with $L C$ shall create other "germs" crossing $L C_{-1}$, whose infinitely many preimages are other new "holes" of divergent trajectories.

From Figure $2 \mathrm{~b}$ we can see that $H_{-1}$ is approaching the region $Z_{3}$ from below and, as the parameter $c$ is increased, a new contact bifurcation between $\partial \mathcal{B}(\infty)$ and the critical curve $L C$ is expected to occur. And in fact the same kind 
of bifurcation occurs, now with a contact on the branch $L$ of $L C$, which causes the crossing of this critical curve by the basin $\mathcal{B}(\infty)$ and a portion denoted by $S_{0}$ in Figure 2d appears. The rank-1 preimages of this portion $S_{0}$ create a new "hole" of $\mathcal{B}(O)$ (or "island" of $\mathcal{B}(\infty)$ ) given by $S_{-1}=$ $T_{(1)}^{-1}\left(S_{0}\right) \cup T_{(3)}^{-1}\left(S_{0}\right)$ which are connected through a segment of the critical line $L_{-1}$ (see Fig. 2d). The third rank-1 preimage of $S_{0}, T_{(2)}^{-1}\left(S_{0}\right)$ is on the right of $L_{-1}^{\prime}$, in the grey region. Clearly $S_{-1}$ has further preimages of any rank, and since $S_{-1}$ is completely included in $Z_{1}$ (as in the previous case), its preimages are given by $T_{(2)}^{-k}\left(S_{-1}\right)$, for $k=1,2, \ldots$, and all are in the region $Z_{1}$, and are approaching the Poincaré Equator.

We may expect that a different effect would be associated with a similar contact bifurcation between $\partial \mathcal{B}(\infty)$ and $L C$, but giving rise to a new hole crossing $L C_{-1}$ inside the strip $Z_{3}$ (instead of $Z_{1}$ as in the previous two bifurcations). Indeed this can be seen in the next bifurcation occurring as $c$ is slightly increased. In Figure $2 d$ we can see that the hole $S_{-1}$ is already quite close to the critical curve $L$ which bounds $Z_{3}$, and a new contact and crossing of $L$ will occur. But now the portion of $L$ close to $S_{-1}$ is the image of a portion of $L_{-1}$ inside $Z_{3}$, so that the crossing will give rise to a region, say $Q_{0}$, whose preimages $T_{(1)}^{-1}\left(Q_{0}\right) \cup T_{(3)}^{-1}\left(Q_{0}\right)$ create a new hole $Q_{-1}$ belonging to the strip $Z_{3}$. This is different from the previous cases because now $Q_{-1}$ has, in its turn, three distinct rank-1 preimages, each of which has one or three preimages, and so on. It is clear that a contact bifurcation giving a new hole in $Z_{3}$ shall cause a drastic increase in the number of connected components of $\mathcal{B}(\infty)$ inside the white area. An example is shown in Figure 2e.

Also the "reverse bifurcations" in holes may occur, i.e., holes in $Z_{3}$ which cross $L C$ and enter $Z_{1}$, causing the merging of their preimages (through a contact on $L C_{-1}$ ), and producing a decrease in the number of holes but increasing the dimension of the holes. These bifurcations further modify the basin, creating wider connected components, which can be seen in Figure 2f. Thus all these kinds of contact bifurcations lead to a more and more complex structure of the basin $\mathcal{B}(\infty)$. In Figure $2 \mathrm{f}$ we see that the basin boundary of the fixed point, which is still locally stable, has a fractal structure and from a global point of view (for example with respect to the Lebesgue measure) the basin is quite a deal smaller than the simply connected one represented in

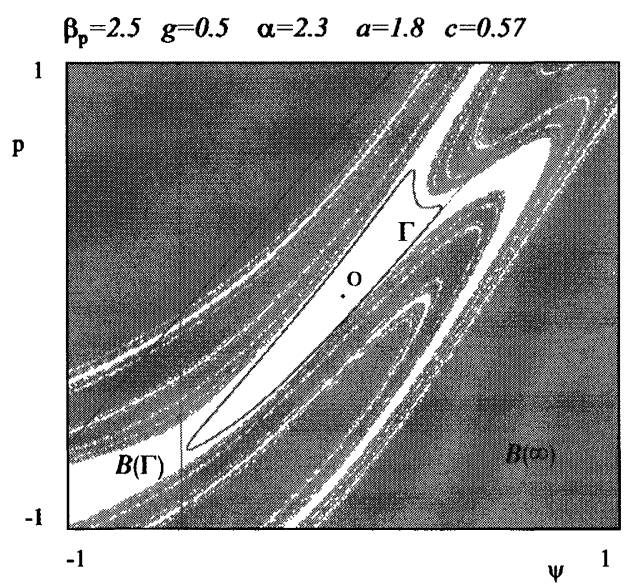

(a)

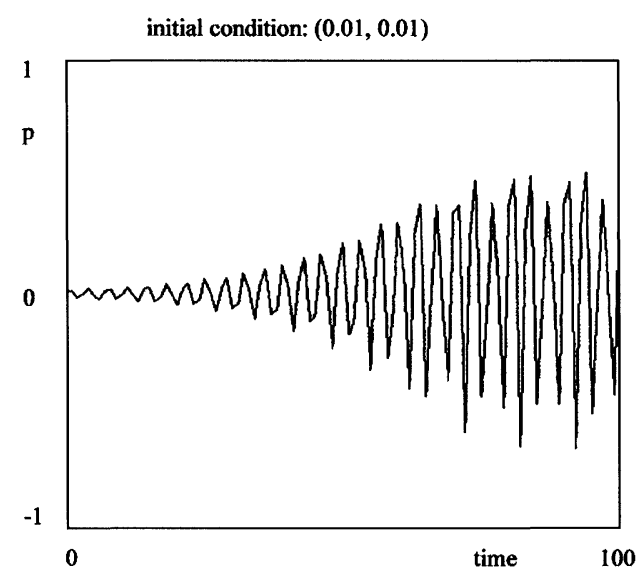

(b)

FIGURE 3 With the same values of the parameters as in Figure $2, \beta_{p}=2.5, g=0.5, \alpha=2.3, a=1.8$, and by increasing $c$ further, the crossing of the Neimark-Hopf bifurcation curve is observed for $c=0 . \overline{5}$, which gives rise to an attracting closed invariant curve $\Gamma$, around the unstable focus $O$, represented together with its basin $\mathcal{B}(\Gamma)$ in (a), obtained with $c=0.57$. The structure of the basin $\mathcal{B}(\Gamma)$, which includes the whole area bounded by $\Gamma$, is the same as the one of the basin $\mathcal{B}(O)$ in Figure $2 \mathrm{f}$, and the dynamic effect of this local bifurcation is simply that of replacing the convergence to $O$ with an oscillatory motion, as shown in (b), where the state variable $p$ is represented as a function of time. 


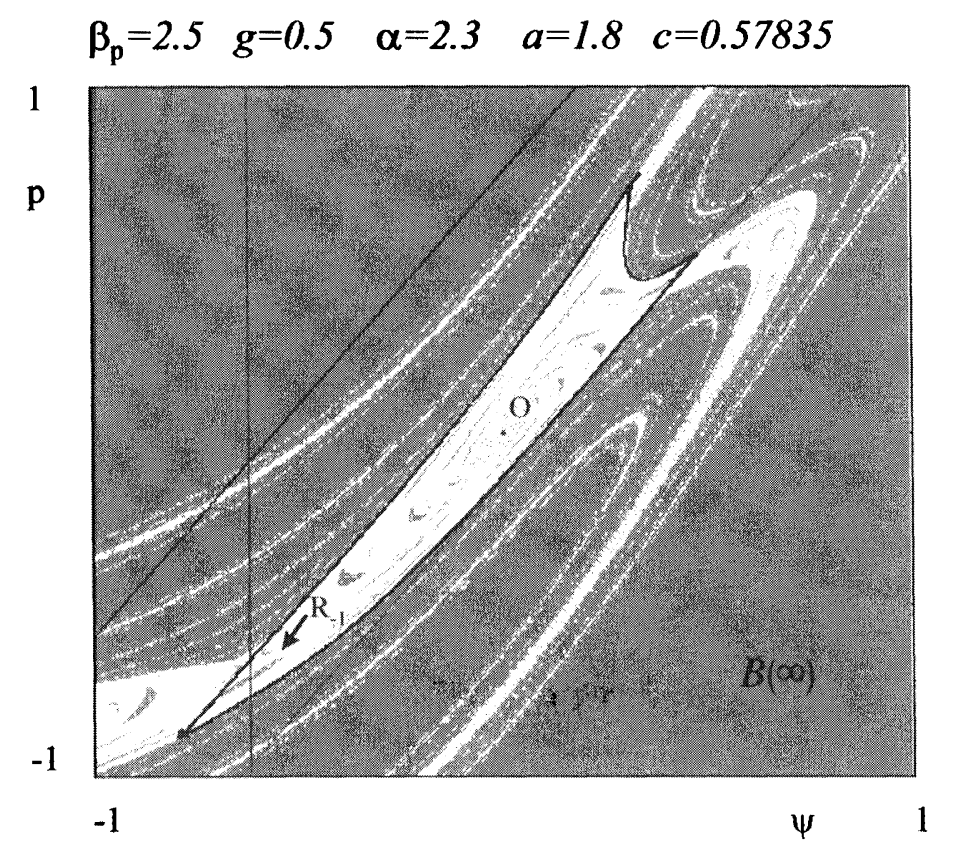

(a)

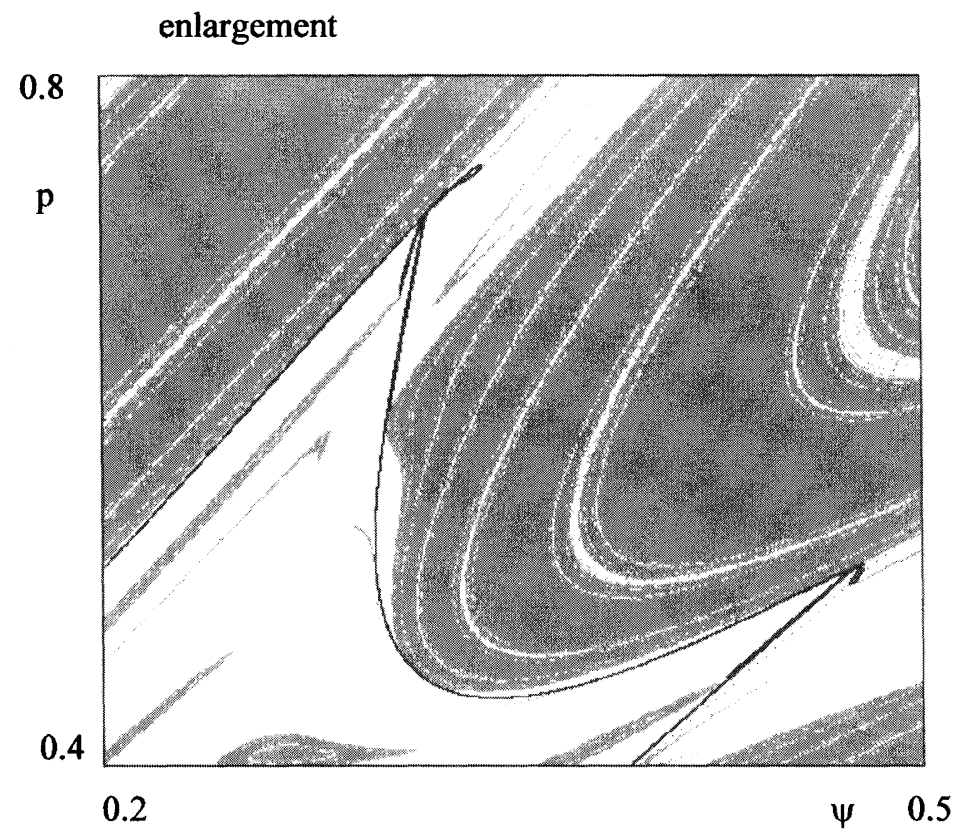

(b)

FIGURE 4 Basin bifurcations observed for the same parameter values as in Figure 3, $\beta_{p}=2.5, g=0.5, \alpha=2.3, a=1.8$, by further increasing $c(c=0.57835)$. Holes are now also inside the area bounded by the attracting set (a). Figure (b) is an enlargement of (a), and shows that the attractor is no longer a closed invariant curve homeomorphic to a circle.

Figure 2a. It is clear that if we allow possible exogenous shocks which can move the state points, as in a "true dynamic state", we can easily say that such a stable regime of $O$ is almost unstable, because nearby states belong to $\mathcal{B}(\infty)$. 
It may be interesting to investigate the dynamic behaviour of $T$ also for higher values of $c$, although we know that it is an almost unstable regime from an applied point of view.

As $c$ crosses the bifurcation value on the Neimark-Hopf curve $(c=0 . \overline{5})$, a supercritical bifurcation occurs, giving rise to a closed invariant attracting curve, say $\Gamma$, around the unstable focus $O$. Roughly speaking we can say that the "old basin" $\mathcal{B}(O)$ changes into the basin $\mathcal{B}(\Gamma)$, an example of which is shown in Figure $3 \mathrm{a}$. In that figure it is evident that, although the frontier $\partial \mathcal{B}(\Gamma)$ has a complex structure, the basin $\mathcal{B}(\Gamma)$ includes the whole area bounded by the curve $\Gamma$. That is, any initial condition close to the fixed point, now an unstable focus, will have a trajectory asymptotic to $\Gamma$, that is the convergent states are now replaced by an oscillatory motion, as shown in Figure $3 \mathrm{~b}$.

But the contact bifurcation of the type described above may change further the basin $\mathcal{B}(\Gamma)$. In fact, a crossing of $\mathcal{B}(\infty)$ through $L$ may give rise to a new hole crossing $L_{-1}$ in the portion inside the area bounded by $\Gamma$, as the new hole $R_{-1}$ in the example shown in Figure 4a at a higher value of $c$. It is clear that now, since $R_{-1}$ is in $Z_{3}$, more and more holes are created, but the new peculiarity is that the infinite sequence $T_{(1)}^{-k}\left(R_{-1}\right), k=1,2, \ldots$, must necessarily spiral towards the fixed point $O$, thus infinitely many holes now exist also in the area bounded by the attracting set. This means that in any neighbourhood of the fixed point there exists a set of positive measure of points having divergent trajectories. As it is evident in the enlargement of Figure $4 b$, the attracting set in Figure 4a is no longer a closed invariant curve $\Gamma$, homeomorphic to a circle, as was the set in Figure 3a. In fact, as often occurs, the closed invariant curve $\Gamma$ undergoes a sequence of bifurcations. Besides quasiperiodic orbits as in Figure 3a, several "periodic windows" are observed as $c$ is increased. For example, an attracting cycle of period 13 exists for $0.576<c<0.5768$, and several periodic windows can be observed which alternate to the quasi-periodic regimes. A particular one, an attracting cycle of period 16 , is shown in Figure 5a, which undergoes the usual flip-bifurcations leading to chaotic motion. A cycle of double period is shown in Figure $5 \mathrm{~b}$, and cyclical chaotic pieces of period 16 are shown in Figure $5 c$ (an enlargement in Figure 5d shows that the pieces are all disjoint). Then the bifurcation leading from 16 chaotic pieces to one single attractor of annular chaotic shape (as the one already shown in Figure 4 for a slightly increased value of $c$ ) is generally of homoclinic type (probably here it occurs when the stable and unstable sets of the saddle cycle of period 16, which separates the basins for the map $T^{16}$,

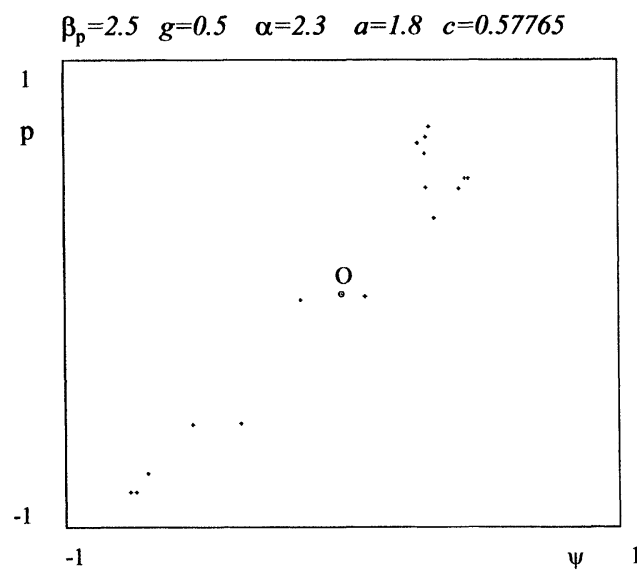

(a)

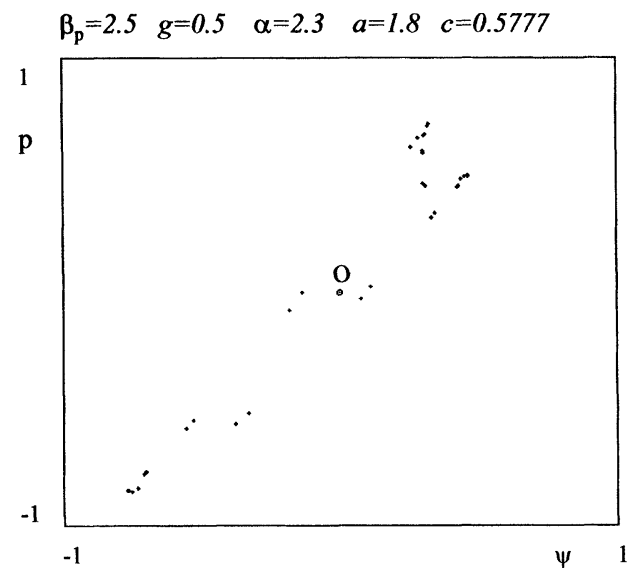

(b)

FIGURE 5 The transformation of the closed invariant curve $\Gamma$ represented in Figure 3 into the attracting set represented in Figure 4 is related to the appearance of "periodic windows" observed as $c$ increases. A particular cycle of period-16 is represented in (a) for $c=0.57765$, which undergoes the usual flip-cascade leading to chaotic motion. Figure (b), obtained for $c=0.5777$, shows a cycle of double period and (c) for $c=0.57781$ shows cyclical chaotic pieces of period-16, which are disjoint, as evidenced in the enlargement in (d). 
$\beta_{p}=2.5 \quad g=0.5 \quad \alpha=2.3 \quad a=1.8 \quad c=0.57781$
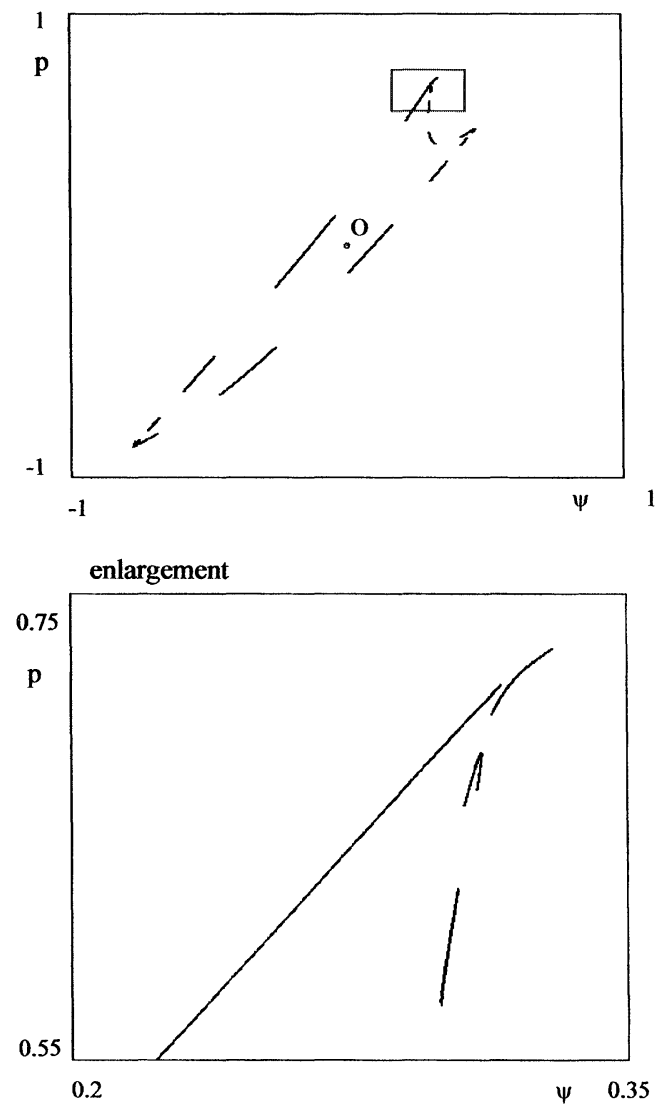

FIGURE 5 (Continued).

intersect one another), and gives rise to attracting sets also called "weakly chaotic rings" in $[16,27]$. Such kinds of homoclinic bifurcation ${ }^{6}$ related to chaotic sets, lead to attracting sets with a complex structure: another example is obtained in Figure 6 in a different regime of parameters.

We remark that the complex attractor observed here, although typical, is not the only possible destiny of a closed invariant curve $\Gamma$. In the next section we shall see that the disappearance of $\Gamma$ may also occur by a homoclinic bifurcation not related to chaotic sets. $\beta_{\mathrm{p}}=3 \quad g=0.3 \quad \alpha=2.75 \quad a=1.5 \quad c=0.4725$

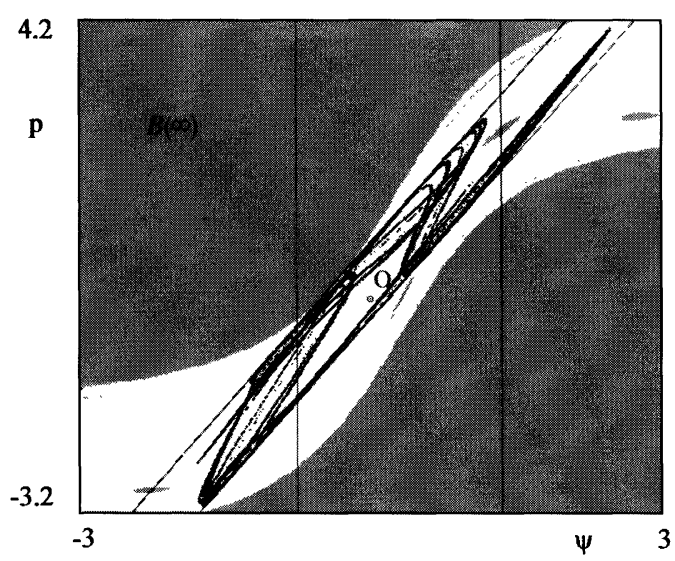

FIGURE 6 A different example of a chaotic attractor of annular shape, obtained for $\beta_{p}=3, g=0.3, \alpha=2.75, a=1.5$, $c=0.4725$ : the basin of the attractor is the white area, while the grey points have divergent trajectories. The presence of only few "holes" is due to the fact that arcs on the boundary of $\mathcal{B}(\infty)$ have "just" crossed the critical curve $L C$.

We close this section noticing that if we fix lower values of $a$, then the Neimark-Hopf bifurcation curve is crossed at lower values of $c$ (as it can be seen from Fig. 1b) so that in the regime of $c$-values in which the basin of bounded trajectories $\mathcal{C}(\mathcal{B}(\infty))$ is simply connected we can see the changes in the attracting set, from a stable focus, to a closed curve $\Gamma$, to a chaotic ring or a chaotic annular area, and the first contact bifurcation between $\partial \mathcal{B}(\infty)$ and $L C$ may occur when the attracting set is any one of these. In the example shown in Figure 6 the first island of $\mathcal{B}(\infty)$ is created when the attractor is already a wide chaotic area.

\section{HOMOCLINIC CONNECTION AND DISAPPEARANCE OF $\Gamma$}

It is well known that the theorem associated with a pair of complex eigenvalues, known as Neimark-Hopf, is only valid locally. Thus the crossing of a Neimark-Hopf bifurcation curve of

\footnotetext{
${ }^{6} \mathrm{We}$ recall that a homoclinic orbit whose points come from the transverse intersection of the stable and unstable sets of a cycle has the peculiar property (see [32, 20], and the references therein) that in any neighbourhood of the homoclinic orbit there exists a closed invariant Cantor set $\Lambda$ on which the restriction of the map is chaotic in the sense of Li and Yorke (see [23]), that is, the map has the same properties as the shift map.
} 
supercritical type proves the existence of an attracting closed curve, say $\Gamma$, only for a "small" range of values of the parameter which is allowed to vary (considering the map as a function of one parameter). What is the fate of such a closed curve $\Gamma$ (whose existence is associated with oscillating behaviour near the fixed point) is an open problem. Its appearance and disappearance is related to the kind of noninvertibility of the map, and often associated with global bifurcations. In Section 3 we have already seen one of the typical mechanisms causing its destruction (as a closed invariant set homeomorphic to a circle), that is, the appearance of a particular cycle leading, via a sequence of bifurcations (flip bifurcations and homoclinic bifurcations), to a chaotic set of annular shape. In this section we shall see a second kind of mechanism leading to the destruction of $\Gamma$, which is associated with a homoclinic connection, not related to chaotic dynamics. Let us consider a different parameter set (always giving an example belonging to case $\mathrm{B}$, i.e., $\beta_{p} k^{\prime}(0)>1$, as in the previous section), fixing $\beta_{p}=1.2, g=0.5, \alpha=1.2, a=2.5$, and increasing the parameter $c$ in the stability region.

Now the interval of $c$-values in which the fixed point is stable is wider, the Neimark-Hopf bifurcation occurs at $c \simeq 0.95178$, and the fractalization of the basin does not occur in the range $c<1$. However, instead of the divergent trajectories, a second attractor appears. That is, we shall see that the basin $\mathcal{B}(O)$ shares the phase plane of interest (given by the complementary set of $\mathcal{B}(\infty)$, i.e., the set of bounded trajectories) with a coexisting attractor, namely a cycle of period three, labelled $\mathcal{C}_{3}{ }^{7}$

In the case shown in Figure 7a, the fixed point $O$ is still the only attractor. The dark grey points belong to $\mathcal{B}(\infty)$ and its complementary set is $\mathcal{B}(O)$, in which we distinguish the white region, given by the points whose trajectories enter a neighbourhood of $O$ of radius $r=0.001$ in less than 300

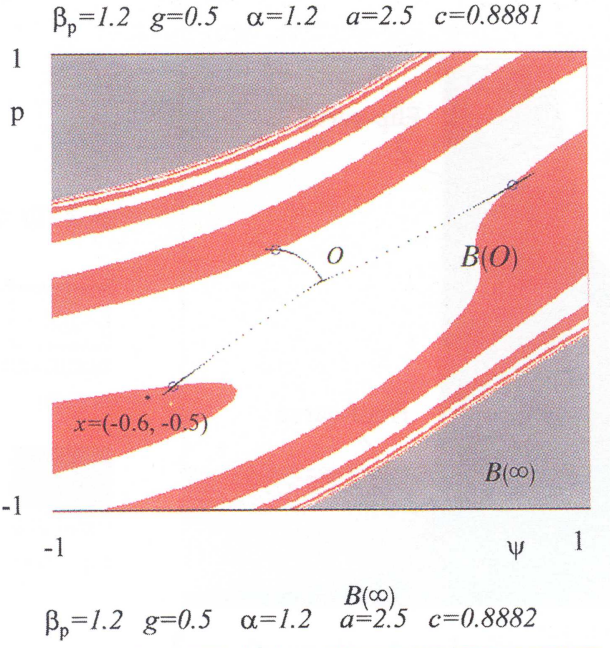

(a)

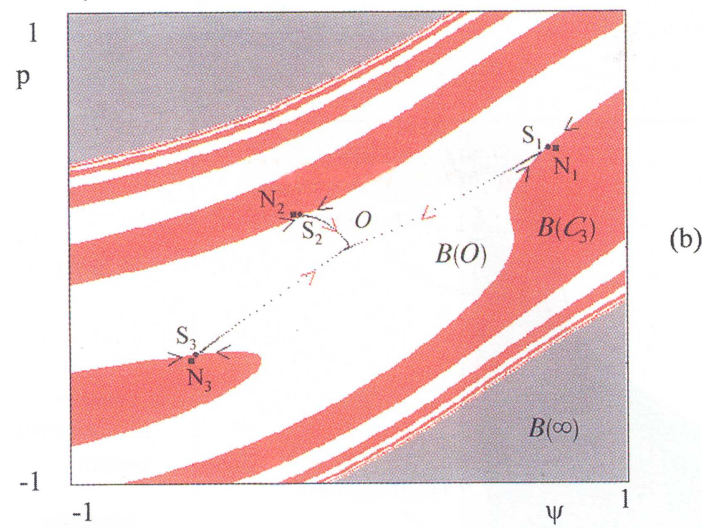

FIGURE $7 \beta_{p}=1.2, g=0.5, \alpha=1.2, a=2.5$. In (a), for $c=0.8881$, the fixed point is the only attractor, with a wide and connected basin, in which the white region is given by the points whose trajectories enter a neighbourhood of $O$ of radius $r=0.001$ in less than 300 iterations, while the red area is made up of the points which converge to $O$ more slowly. A trajectory starting in the red region moves cyclically for a long time inside the three balls, before converging to $O$ (intermittency) and this behaviour anticipates the appearance, via saddle-node bifurcation, of a 3-cycle attracting node $\left\{N_{1}, N_{2}\right.$, $\left.N_{3}\right\}$ and a 3-cycle saddle $\left\{S_{1}, S_{2}, S_{3}\right\}$, which are represented in (b) for $c=0.8882$. The red area in (b) represents the basin of the attracting 3-cycle $\mathcal{C}_{3}=\left\{N_{1}, N_{2}, N_{3}\right\}$, while the white area is the basin of the attracting fixed point $O$. The basin of the attracting node $\mathcal{C}_{3}$ may be subdivided further into three different regions, by considering the periodic points as fixed points of the map $T^{3}$, as shown in (c), obtained for $c=0.95$. From the same figure we see that the competing attracting set $\mathcal{C}_{3}$ attracts now most of the points with bounded trajectories. (See Color Plate II.)

\footnotetext{
${ }^{7}$ We recall that for a two-dimensional map the existence of a periodic orbit of period three is not related to the existence of chaotic dynamics, as it occurs in one-dimensional maps. This example is precisely a demonstration. In the case shown in Figure $7 \mathrm{~b}$ the $\Omega$-limit set of $T$ (which includes the limit set of all the trajectories of the plane) includes only a few cycles of $T$.
} 


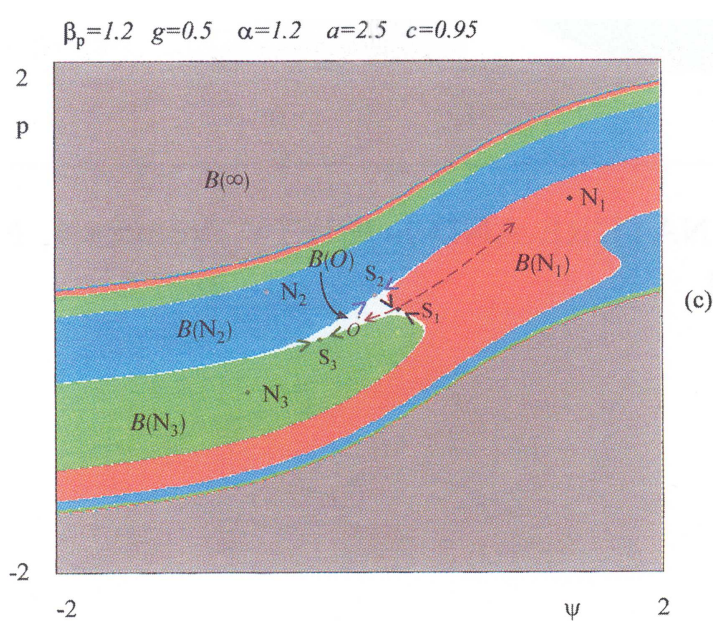

FIGURE 7 (Continued).

iterations. Also the other points (the red area) converge to $O$, but in a greater number of iterations. A trajectory starting in the point $(-0.6,-0.5)$ is shown, which reveals three balls in which the points are more dense (see Fig. 7a). The trajectory cycles for a long time between points in these balls before finally converging to $O$. This behaviour (also known as intermittency) anticipates the appearance, via saddle-node bifurcation, of cycles of period-3.

In fact, at a value of $c$ just slightly increased, two different cycles of period-3 exist: an attracting node $\mathcal{C}_{3}=\left\{N_{1}, N_{2}, N_{3}\right\}$, whose basin $\mathcal{B}\left(\mathcal{C}_{3}\right)$ is shown as the red area in Figure $7 b$, and a 3-cycle saddle whose points $S_{1}, S_{2}, S_{3}$ belong to the boundary of the immediate basin of the attracting 3-cycle (and are very close to the points of the attracting cycle $\mathcal{C}_{3}$ in Fig. 7b). For the map $T^{3}$ all the points $N_{i}, i=1,2,3$, are fixed points, ${ }^{8}$ attracting nodes, and the boundary of their basin is made up of the stable sets of the saddles $S_{i}, i=1,2,3$, and separates the basins $\mathcal{B}\left(N_{i}\right), i=1,2,3$, from the basin $\mathcal{B}(O)$.

In fact (see Fig. $7 \mathrm{c}$ at a higher value of $c$ ) for any $i$, $i=1,2,3$, the stable set $W^{s}\left(S_{i}\right)$ gives the boundary $\partial \mathcal{B}\left(N_{i}\right)$, while the two unstable branches $W^{u}\left(S_{i}\right)$ go towards $N_{i}$ from one side (entering the coloured basins) and towards the fixed point $O$ from the other side (entering the white area $\mathcal{B}(O)$ ).

In this configuration of the basin structure we have the crossing of the Neimark-Hopf curve in the plane $(c, a)$ of the parameters, which gives rise to a closed invariant curve $\Gamma$. The basins are shown in Figure 8a, and we can see that the structure is the same as that of Figure $7 \mathrm{c}$ except that the white area now denotes $\mathcal{B}(\Gamma)$ instead of

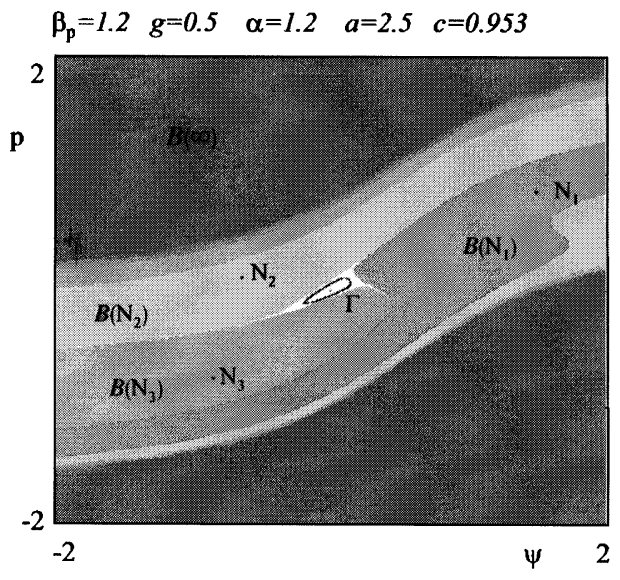

(a)

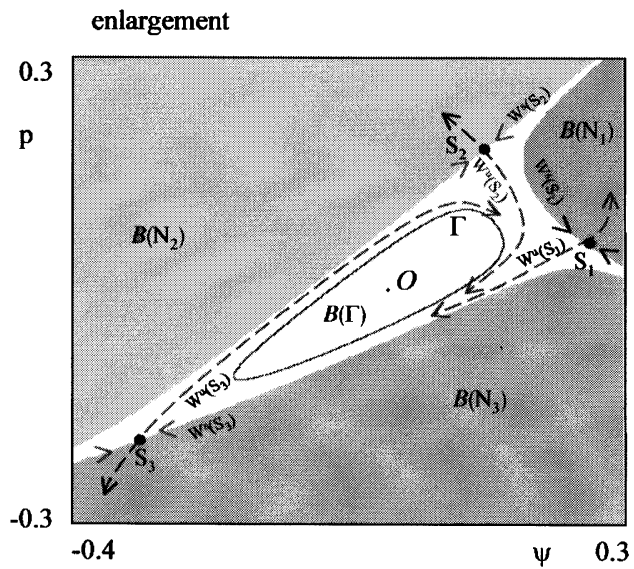

(b)

FIGURE 8 Starting from Figure $7 \mathrm{c}$, where $\beta_{p}=1.2, g=0.5$, $\alpha=1.2, a=2.5, c=0.95$, the crossing of the Neimark-Hopf bifurcation curve observed by slightly increasing $c$ leads to the creation of a closed invariant curve $\Gamma$, represented in (a) for $c=0.953$. The enlargement in (b) shows that the basin of $\Gamma$ is bounded by the stable sets $W^{s}\left(S_{i}\right)$ of the saddle points $S_{i}$, $i=1,2,3$, while the unstable set $W^{u}\left(S_{i}\right)$ of each saddle $S_{i}$ has one branch converging to $N_{i}$ and the other converging to $\Gamma$. (See Color Plate III.)

\footnotetext{
${ }^{8}$ From now on we will speak, indifferently, of $k$-cycles of $T$ or fixed points of $T^{k}, k=2,3, \ldots$
} 
$\mathcal{B}(O)$. In the enlargement of Figure $8 \mathrm{~b}$ we can see the structure of the stable and unstable sets of $S_{i}$, $i=1,2,3$. Note that now the unstable set of $S_{i}$, $i=1,2,3$, goes on one side to $N_{i}$ and on the other side to $\Gamma$, converging to $\Gamma$ from the outside of the curve. As often occurs, as $c$ increases the area enclosed by $\Gamma$ increases, i.e., $\Gamma$ becomes wider. In Figure $8 \mathrm{~b}$ we can see that $\Gamma$ is approaching the boundary of its basin, and in fact a global bifurcation is about to occur, which shall cause the destruction of the curve $\Gamma$ (and thus of the oscillating, quasi-periodic behaviour around $O$ ). This can be seen in Figure 9a, at a very close value of $c$ : the curve $\Gamma$ disappears and the 3 -cycle $\mathcal{C}_{3}$ is the only surviving attractor; this cycle corresponds to three fixed points for the map $T^{3}$ whose basins are shown in Figure 9a, together with a trajectory starting very close to the repelling focus $O$. The enlargement in Figure $9 \mathrm{~b}$ clearly shows that the old "white area" constituting $\mathcal{B}(\Gamma)$ is now shared between the three fixed points $N_{i}$ of $T^{3}$, whose basins are still bounded by the stable sets of the saddles $S_{i}, i=1,2,3$. Something has changed in the behaviour of the unstable set emanating from each $S_{i}, i=1,2,3$ : for instance, the unstable branches issuing from $S_{2}$ are going to $N_{2}$ from one side and to $N_{1}$ to the other side. We can also see from Figure $9 \mathrm{~b}$ that $W^{s}\left(S_{2}\right)$ now separates $\mathcal{B}\left(N_{2}\right)$ from $\mathcal{B}\left(N_{1}\right)$, and one branch of the stable set $S_{2}$ emanates, spiralling, from the origin. The same occurs for the other saddle points (being of cyclical behaviour for the map $T$ ).

The global bifurcation which causes the destruction of $\Gamma$ is called a "homoclinic connection" or "saddle connection" of the saddle cycle $\left\{S_{1}, S_{2}, S_{3}\right\}$ and it is not related to complex dynamics (because it does not give rise to transverse intersection between the stable and unstable sets of the saddle). The qualitative behaviour associated with this global bifurcation is shown in Figure 10. Let us call $c_{\Gamma}\left(c_{\Gamma} \simeq 0.954\right)$ the bifurcation value which causes the disappearance of $\Gamma$, that is, for $c$ close to $c_{\Gamma}$ and $c<c_{\Gamma}$ (resp. $c>c_{\Gamma}$ ) the invariant curve $\Gamma$ still exists (no longer exists). Consider the behaviour of the stable and unstable sets of the saddle

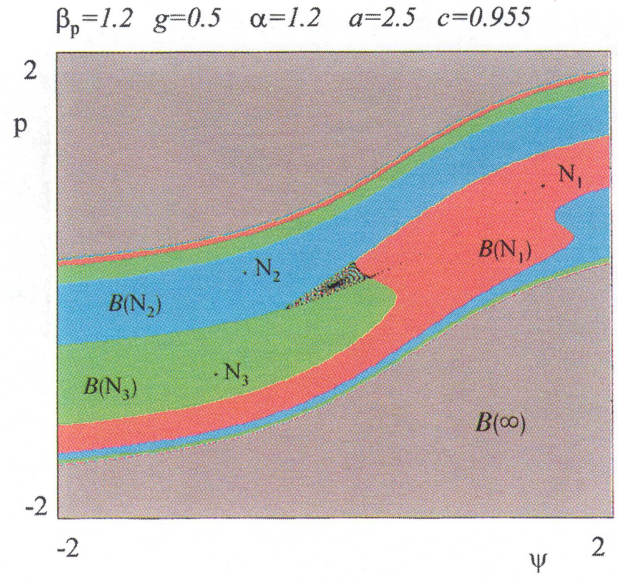

(a)

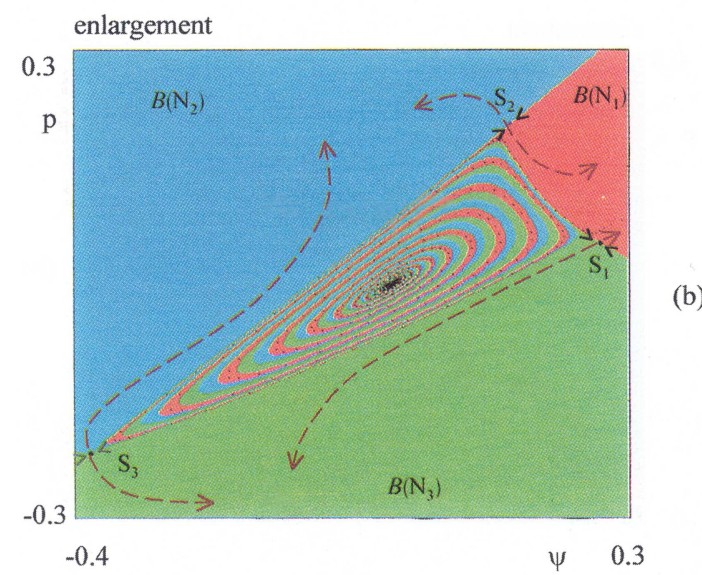

FIGURE 9 With the parameters of Figure 8, by further increasing $c, \Gamma$ increases in size approaching the boundary of its basin and a global bifurcation causes the disappearance of $\Gamma$ leaving $N_{1}, N_{2}$, and $N_{3}$ as the only surviving attractors of $T^{3}$, shown in (a), obtained for $c=0.955$, where also a trajectory starting near $O$ is represented, converging to $N_{1}$. The basins of $N_{1}, N_{2}$, and $N_{3}$ now share the old white area (see the enlargement in (b)). It is also shown in (b) that these basins are still bounded by the stable sets of the saddle points, while the two branches of the unstable set of each saddle converge to two different attracting nodes. (See Color Plate IV.)

point $S_{1}$ (the same also holds for the other points by cyclically changing the indexes). In Figure 10a, before the bifurcation, when $\Gamma$ exists, the stable set $W^{s}\left(S_{1}\right)$ is the only one which gives $\partial \mathcal{B}\left(N_{1}\right)$ and both the branches of $W^{s}\left(S_{1}\right)$ come from far away. One of the unstable branches of $S_{1}$ goes to $N_{1}$ and the other to $\Gamma$.

At the bifurcation value $c=c_{\Gamma}$ (see Fig. 10b), the closed invariant curve $\Gamma$ no longer exists, or, we 


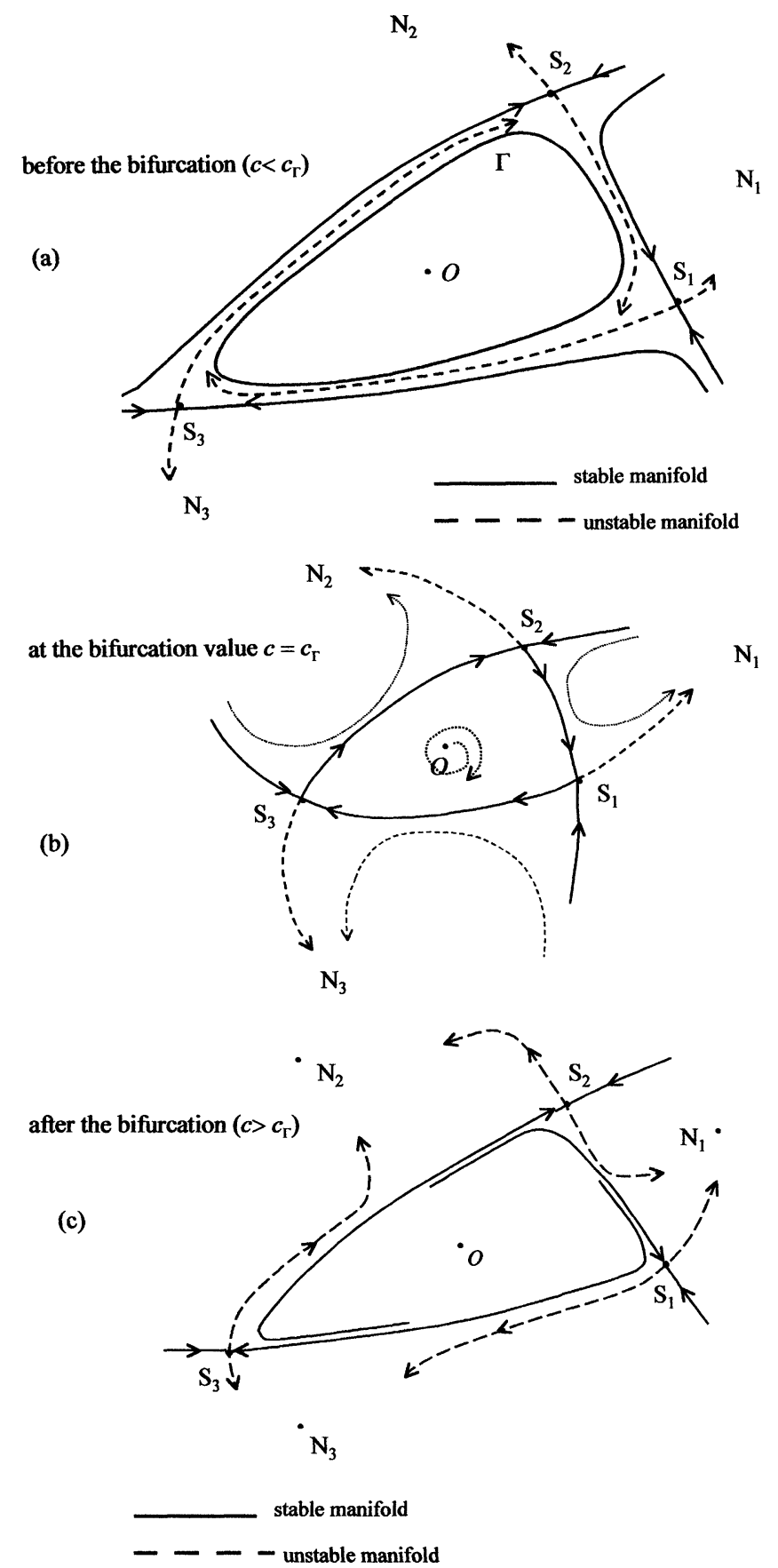

FIGURE 10 Qualitative sketches of the behaviour associated with the global bifurcation causing the disappearance of $\Gamma$. (a) represents the behaviour of the stable and unstable manifolds of the saddle points $S_{i}, i=1,2,3$, immediately before the bifurcation: the stable sets $W^{s}\left(S_{i}\right), i=1,2,3$, bound the basin of $\Gamma$, while the unstable set $W^{u}\left(S_{i}\right)$ of each saddle $S_{i}$ has one branch converging to $\Gamma$ and the other converging to $N_{i}, i=1,2,3$. (b) shows what happens at the bifurcation value $c_{\Gamma}$ of the parameter $c$, where $\Gamma$ merges with the homoclinic connection given by the union of three heteroclinic trajectories connecting the saddle points: one branch of the stable manifold of $S_{1}$ is also a branch of the unstable manifold of $S_{2}$, and so on cyclically. (c) describes the situation existing soon after the bifurcation: $\Gamma$ has disappeared and the only surviving attractors of $T^{3}$ are $N_{1}, N_{2}$, and $N_{3}$, whose basins now share the old basin of $\Gamma$. The boundary of the basin of each node $N_{i}, i=1,2,3$, now includes two saddles, together with their stable sets. For each saddle point $S_{i}, i=1,2,3$, one branch of the stable set $W^{s}\left(S_{i}\right)$ comes from far away, while the other branch emanates, spiralling, from the origin. Also the behaviour of the unstable set emanating from each saddle $S_{i}, i=1,2,3$ is changed. One branch of $W^{u}\left(S_{1}\right)$ converges to $N_{3}$ and the other branch to $N_{1}$, and so on cyclically. 
could say, it merges with the homoclinic connection given by the union of three heteroclinic trajectories, made up of invariant sets connecting the saddle points. That is, one branch of $W^{s}\left(S_{1}\right)$ is also a branch of $W^{u}\left(S_{2}\right)$ and one branch of $W^{u}\left(S_{1}\right)$ is also a branch of $W^{s}\left(S_{3}\right)$ (see Fig. 10b). And so on cyclically.

It is clear that the union of these trajectories connecting $S_{1}, S_{2}$ and $S_{3}$ gives a closed invariant curve, which attracts the trajectories of points inside this area, but repells the trajectories outside. This is the last value in which a closed invariant curve (degeneracy of the attracting $\Gamma$ ) exists.

After the bifurcation (see Fig. 10c, which is a qualitative sketch of Fig. 9b), we have a bifurcation of all the basins: $\mathcal{B}(\Gamma)$ has disappeared and its old area is shared between the three basins $\mathcal{B}\left(N_{i}\right)$, $i=1,2,3$, for the map $T^{3}$. The boundary $\partial \mathcal{B}\left(N_{1}\right)$ now includes two saddles, $S_{1}$ and $S_{2}$, together with their stable sets; one branch of $W^{s}\left(S_{1}\right)$ and one of $W^{s}\left(S_{2}\right)$ come from far away, while the other two branches emanate from the origin.

It is clear that both before and after the bifurcation value $c_{\Gamma}$ the stable and unstable sets of the saddles $S_{i}, i=1,2,3$, never intersect (thus no transverse homoclinic points exist). Only at the bifurcation value $c_{\Gamma}$ do we have a homoclinic connection of the saddle cycle, which is a kind of "homoclinic tangency" not followed by crossing, and thus not related to chaotic behaviour. In fact for the map $T$ we have a unique attractor, the 3cycle $\mathcal{C}_{3}=\left\{N_{1}, N_{2}, N_{3}\right\}$, an attracting node, whose basin is such that $\overline{\mathcal{B}\left(\mathcal{C}_{3}\right)}=\mathcal{C}\left(\mathcal{B}(\infty)\right.$ ) (i.e., $\overline{\mathcal{B}\left(\mathcal{C}_{3}\right)} \cup$ $\mathcal{B}(\infty)=\mathbf{R}^{2}$.

\section{FRACTALIZATION OF BASINS IN INVERTIBLE MAPS AND WADA-BASINS}

In the same example considered above, in Section 4 , for higher values of $c$ there exists either the 3 -cycle $\mathcal{C}_{3}$ as a unique attractor, or a 6 -cycle $\mathcal{C}_{6}$ flip-bifurcated from $\mathcal{C}_{3}$. Although the range of economic interest of the parameter $c$ is mainly restricted to the interval $(0,1]$, we investigate the dynamic behaviour of the map up to $c=1.2$ in order to demonstrate interesting dynamic phenomena.

As we have seen in Section 2.3, for $c>1$ the map becomes invertible, thus the complex structure of the basins is no longer related to global bifurcations of contact with the critical set $L C$, but complex behaviour appears only via the standard mechanisms of homoclinic bifurcations creating transverse intersections ${ }^{9}$ between stable and unstable manifolds of saddles.

An interesting example is related to the case we are here analyzing, because it gives rise to the phenomenon of "Wada-basins", as introduced in $[29,28]$. These are invariant sets, or frontiers, having the property that any neighbourhood of any of their points contains points of at least three different basins.

Let us consider the same example of the previous section at a greater value of $c$ with respect to the one considered in Figure 9. In Figure 11a we show the basins of the map $T^{3}$, for which the attractors are now three cycles of period-2 (i.e., the 6-cycle for $T$, flip-bifurcated ${ }^{10}$ from $\mathcal{C}_{3}$ ). In the enlargement of Figure $11 \mathrm{~b}$ we see that the "tongues" of the basin bounded by the stable set $W^{s}\left(S_{2}\right)$ are approaching one branch of the unstable set $W^{u}\left(S_{2}\right)$. At a value of $c$ in the interval $(1.04,1.05)$ the homoclinic tangency between $W^{s}\left(S_{2}\right)$ and $W^{u}\left(S_{2}\right)$ occurs (and similarly for the other saddles $S_{1}$ and $S_{3}$ ), followed by a transverse intersection, as it can easily be seen in Figures 11c, d, where the "tongues" have now crossed $W^{u}\left(S_{2}\right)$.

This homoclinic bifurcation is related to chaotic behaviour by means of the horse-shoe mechanism, as explained by Smale (see [32,20]), giving rise to the characteristic "tangle" in the neighbourhood of the saddle, as also described in [6] and [14]. This is the standard route to fractal basins in invertible

\footnotetext{
${ }^{9}$ We recall that for invertible maps a basin is said to be fractal if it includes at least one transverse homoclinic point $([30,24,3,19])$

${ }^{10}$ That is, each $N_{i}$ has become unstable giving rise to an attracting 2-cycle.
} 


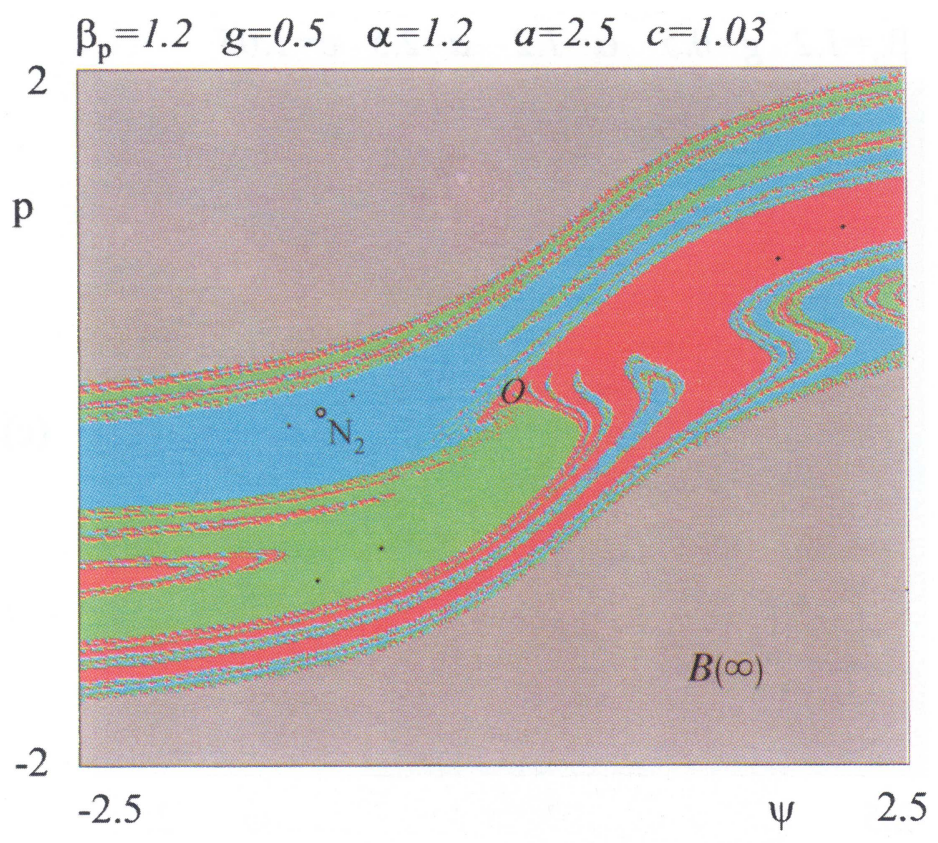

(a)

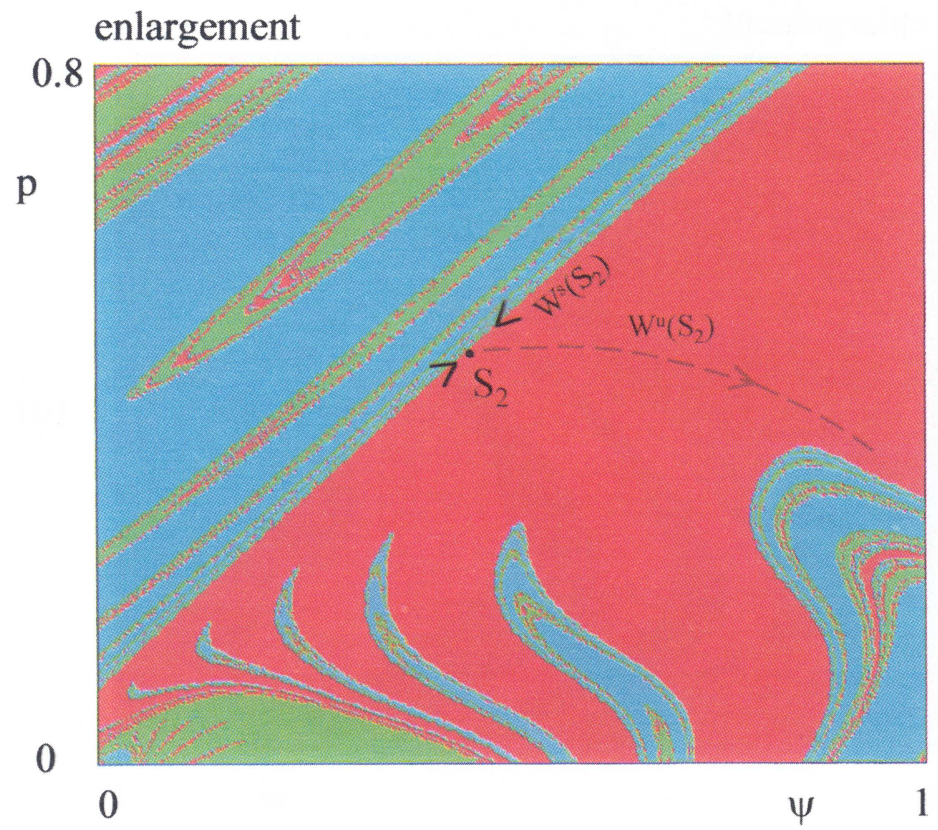

(b)

FIGURE 11 An example of the route to fractal basins, observed in the same regime of parameters considered in Figures 7-9, i.e., $\beta_{p}=1.2, g=0.5, \alpha=1.2, a=2.5$, and for values of $c>1$, in the region of invertibility of the map $T$. For $c=1.03$ the attracting sets of the map $T^{3}$ are three cycles of period-2, flip-bifurcated from the three fixed points represented in Figure 9 , whose basins are represented with different colours in $(a)$. The enlargement in $(b)$ shows that the "tongues" of the basins, bounded by the stable set $W^{s}\left(S_{2}\right)$ of the saddle $S_{2}$ are approaching one branch of the unstable set, $W^{u}\left(S_{2}\right)$. At a value of $c$ in the interval $(1.04,1.05)$ the homoclinic tangency between $W^{s}\left(S_{2}\right)$ and $W^{u}\left(S_{2}\right)$ occurs, followed by a transverse intersection, as shown in $(c)$ and $(d)$, obtained for $c=1.05$. This homoclinic bifurcation gives rise to the characteristic "tangle" in the neighbourhood of the saddle. (See Color Plate V.) 


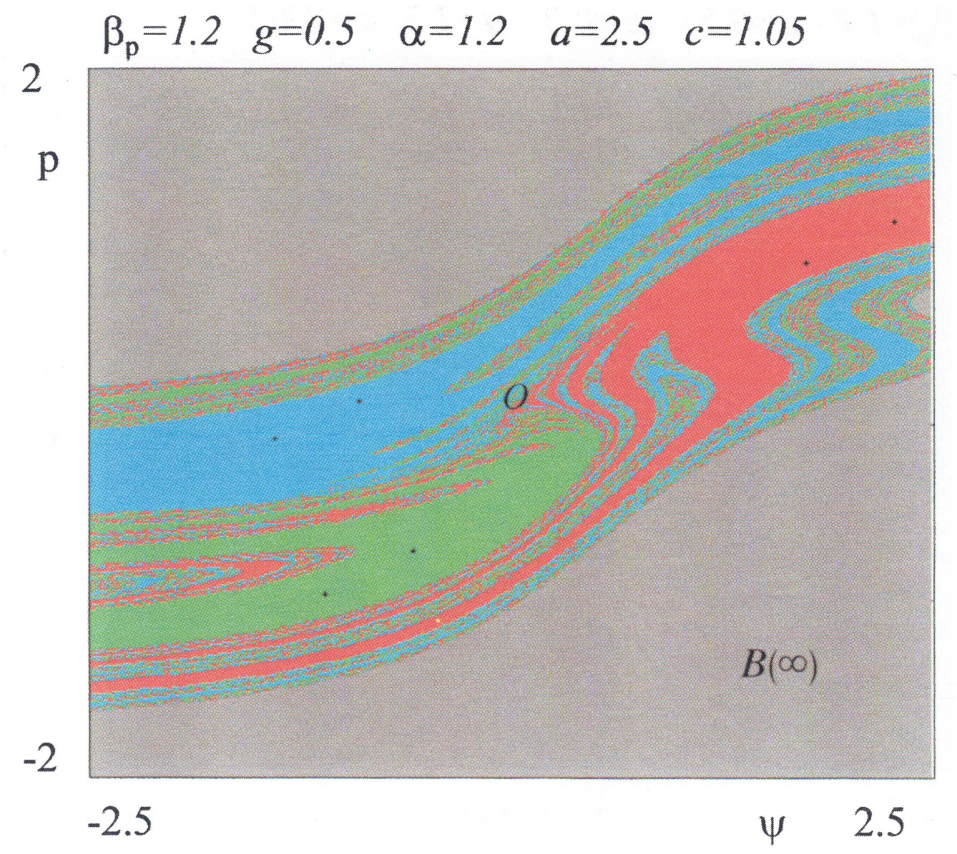

(c)

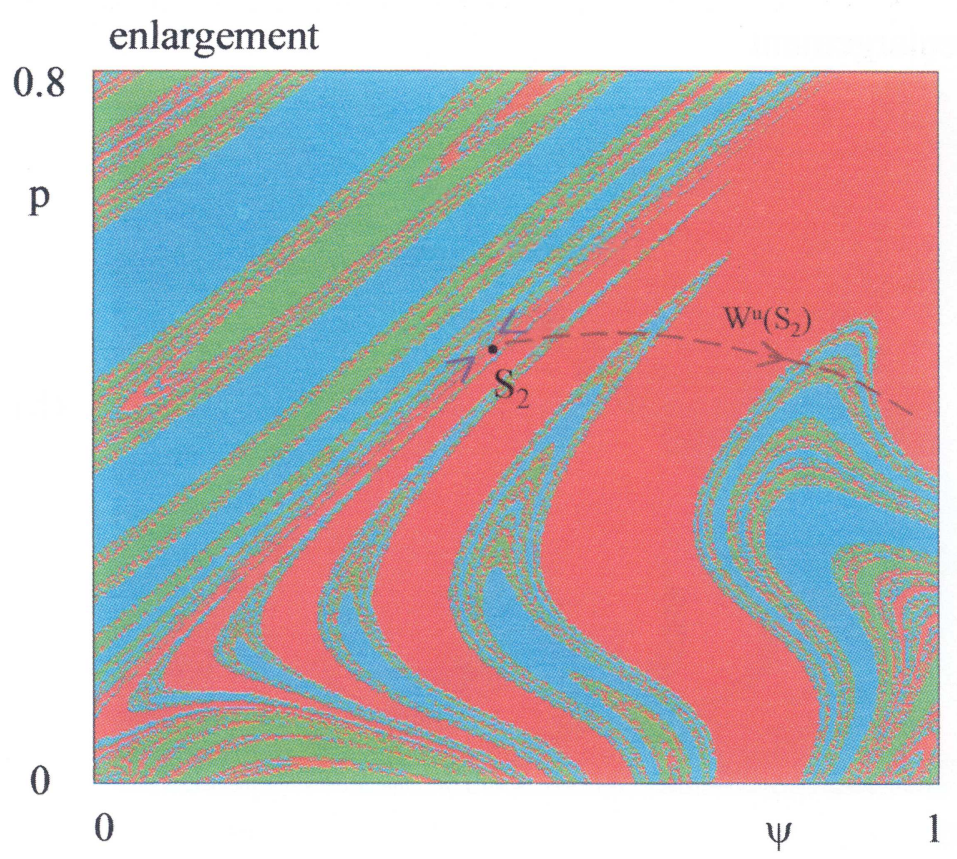

(d)

FIGURE 11 (Continued).

maps. Clearly for the map $T$ the fractal frontier, say $\mathcal{F}$, where the map is chaotic, is almost "invisible". In fact, considering the map $T$, we have that the basin of attraction of the stable 6-cycle $\mathcal{C}_{6}$ is a simply-connected region, the complementary set of $\mathcal{B}(\infty)$, except for the invariant 
set $\mathcal{F}$ of zero Lebesgue measure on which the dynamics of the restriction of $T$ is chaotic. The global behaviour is essentially as shown in
Figure 12a, where only $\mathcal{B}(\infty)$ (the grey region) and $\overline{\mathcal{B}\left(\mathcal{C}_{6}\right)}$ (the white region) are shown. It is well known that the dynamic effect of the existence of

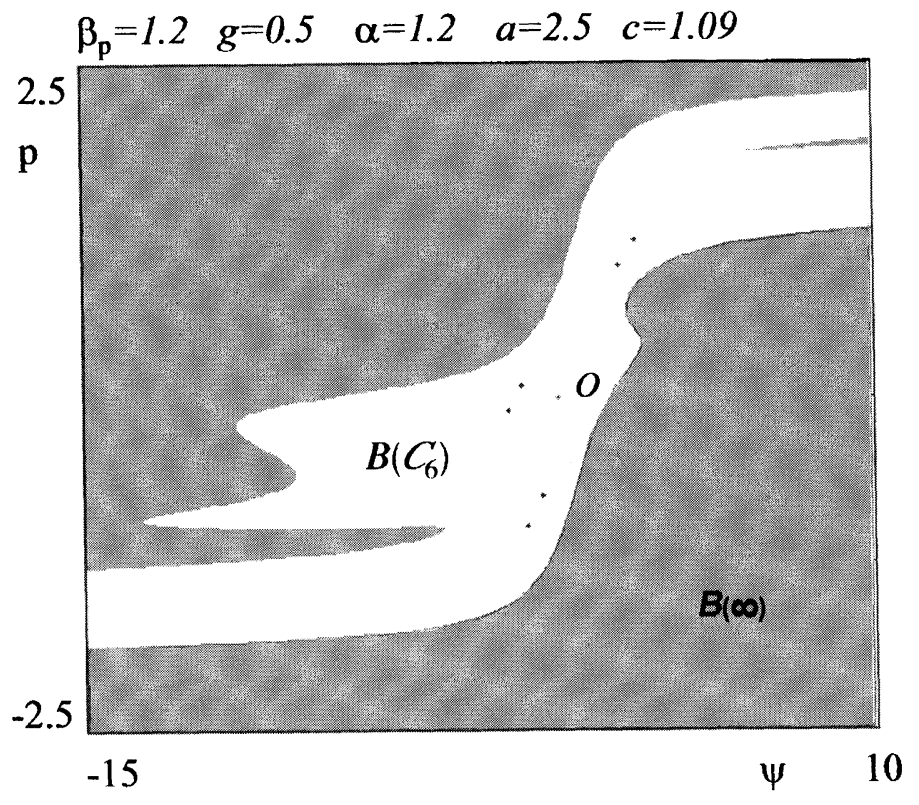

(a)

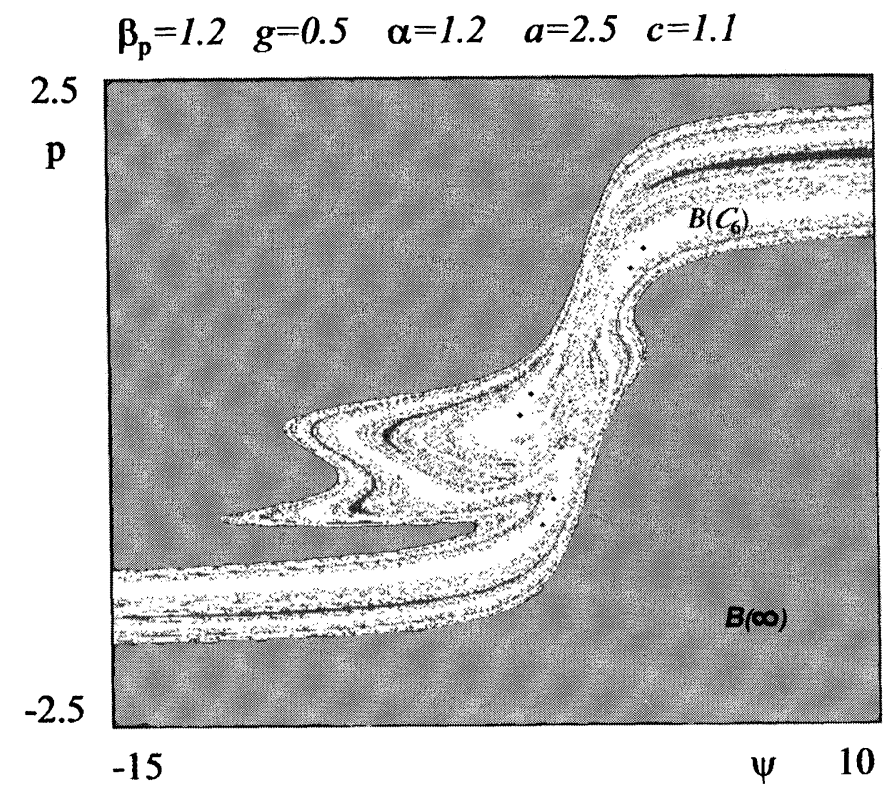

(b)

FIGURE 12 The fractal structure of the basins highlighted in Figure 11 becomes "invisible" if we consider the map $T$ instead of $T^{3}$ and represent the basin $\mathcal{B}\left(\mathcal{C}_{6}\right)$ of the 6-cycle as a whole, as shown in (a), obtained for $\beta_{p}=1.2, g=0.5, \alpha=1.2, a=2.5$ and $c=1.09$. As $c$ increases another homoclinic bifurcation, similar to the one observed in Figure 11, but now involving a saddle cycle belonging to $\partial \mathcal{B}(\infty)$, causes the spread of portions of $\mathcal{B}(\infty)$ inside the old white area, as shown in (b), obtained for $c=1.1$. (c) represents the structure of the basins for the same parameters values, but considering the map $T^{3}$. 


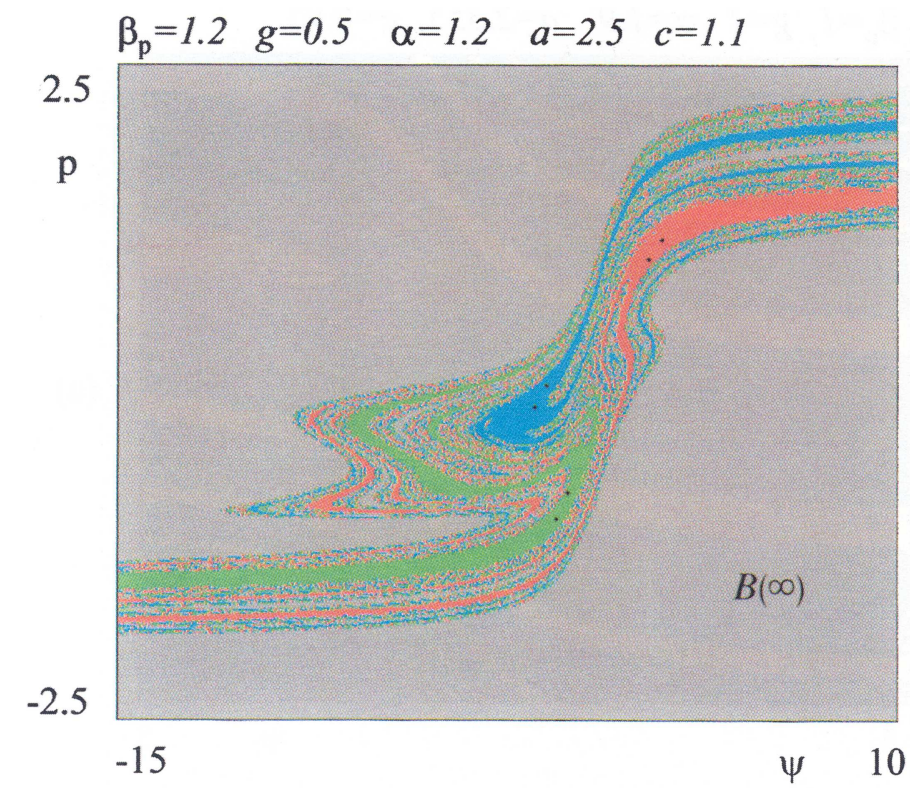

(c)

FIGURE 12 (Continued). (See Color Plate VI.)

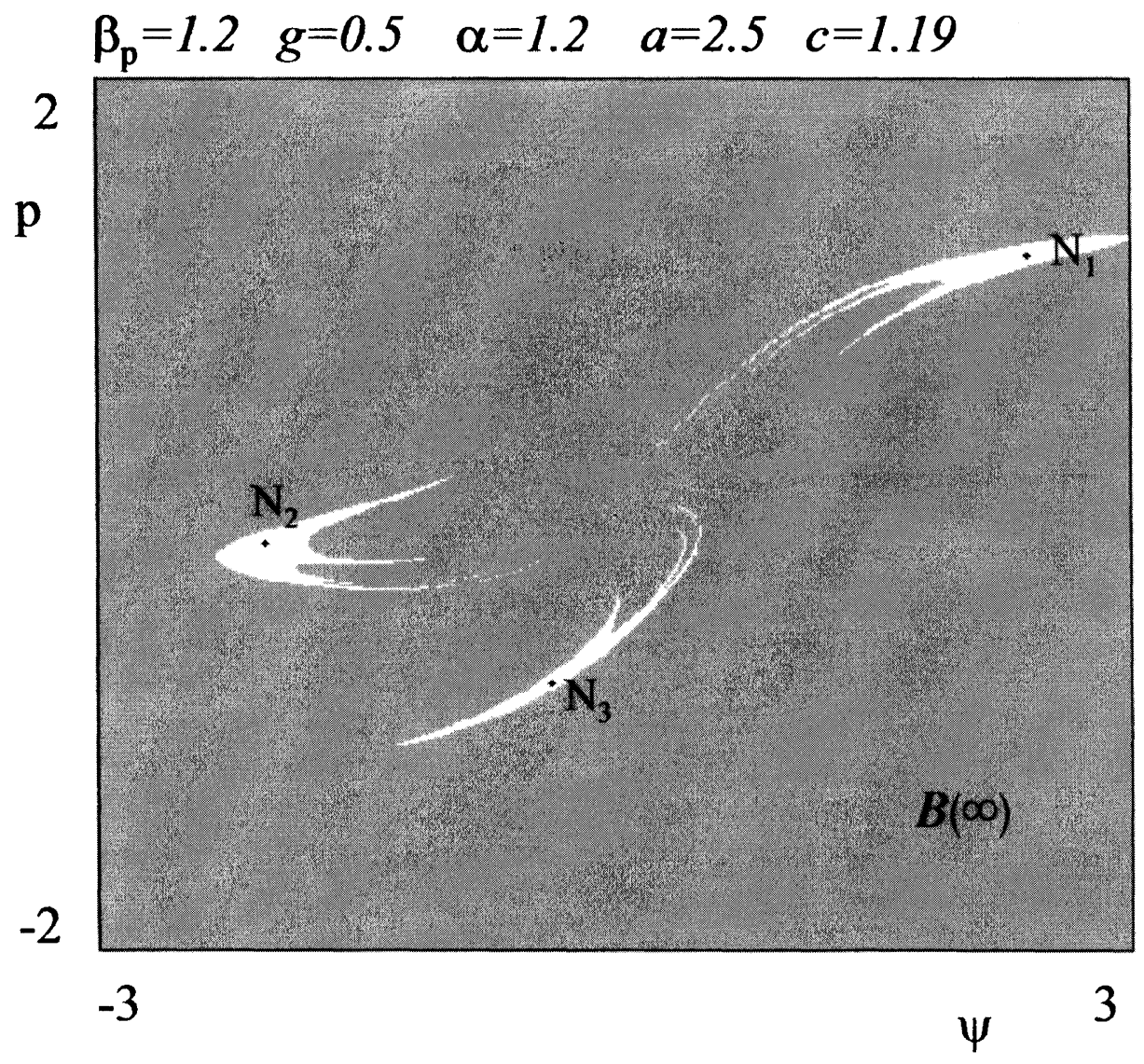

FIGURE 13 The effect of a reverse flip bifurcation observed by increasing $c$ further with respect to the parameter situation of Figure 12. For $\beta_{p}=1.2, g=0.5, \alpha=1.2, a=2.5$ and $c=1.19$, an attracting 3-cycle $\mathcal{C}_{3}$ is again the only attractor, as in Figure 9a, but its basin is now very small as compared to $\mathcal{B}(\infty)$. 
the chaotic invisible set $\mathcal{F}$ is related to the transient behaviours of the trajectories, which may have a chaotic part before converging to the 6-cycle, especially for initial conditions in the chaotic "tangle", near the homoclinic saddles.

As $c$ increases we can observe another homoclinic bifurcation, now associated with a saddle cycle belonging to $\partial \mathcal{B}(\infty)$. The dynamic effect for the map $T$ is more "dangerous" in this case because it causes the spread of portions of $\mathcal{B}(\infty)$ inside the old area of bounded trajectories. An example of the basin structure of $\mathcal{B}(\infty)$ after this homoclinic bifurcation is shown in Figure $12 \mathrm{~b}$. Clearly the region of white points of Figure $12 b$ must further be divided if we consider the map $T^{3}$, as it is shown in Figure 12c. It is worth noting that now the frontier of all the four basins of $T^{3}$ has the peculiar property that in any neighbourhood of any of its points we can find points belonging to four different basins (the attractors are the three 2cycles and a point at infinity). As a final dynamic curiosity of this example let us see what happens if $c$ is further increased: the 6-cycles of $T$ undergoes a reverse flip bifurcation leaving again an attracting 3-cycle $\mathcal{C}_{3}$, whose basin becomes smaller and smaller, being $\mathcal{B}(\infty)$ the dominating one, as shown in Figure 13.

\section{PERSistent COEXISTENCE OF ATTRACTING SETS}

While in Section 3 we have seen a competition between the basin $\mathcal{B}(O)$ of the stable fixed point and the basin of divergent trajectories $\mathcal{B}(\infty)$, and in Section 4 the competition between $\mathcal{B}(O)$ and a coexistent 3-cycle $\mathcal{C}_{3}$ for a short range of $c$-values, in this section we shall see how, considering the map $T$ as a function of the parameter $a$ for a fixed value of $c$, we can observe a persistent competition between $\mathcal{B}(O)$ and the basin $\mathcal{B}(\mathcal{A})$ of a different attracting set $\mathcal{A}$, a stable cycle or a cyclic chaotic set. Let us consider the fixed values $c=0.95$, $\beta_{p}=1, g=1$ and $\alpha=1.9$ and increase $a$, starting from low values in the stability region, whose shape is qualitatively similar to the one represented in Figure 1a (this example being included in case A). We also note that by increasing $a$ the map $T$ always remains in the region of noninvertibility.

At low values of $a$ the origin $O$ seems to be the only attractor with a wide and connected basin, but as $a$ increases the nonlinearity of $T$ brings about saddle-node bifurcations, thereby creating other attractors, coexistent with $O$. A "periodic window" associated with a stable 4cycle $\mathcal{C}_{4}$ is obtained in the range given approximately by $a \in[1.775,2]$. In Figures $14 \mathrm{a}, \mathrm{b}$ we see how the basin of bounded trajectories is shared between $\mathcal{B}(O)$ and $\mathcal{B}\left(\mathcal{C}_{4}\right)$. Two 4-cycles appear via a forward saddle-node bifurcation at $a \simeq 1.775$, one attracting node and one saddle, whose stable set belongs to the basin boundaries for the map $T^{4}$. As the parameter $a$ increases, a backward saddle-node bifurcation causes the merging and disappearance of the two cycles at $a \simeq 2$, leaving again the origin as the only attractor of bounded trajectories. As $a$ is further increased another saddle node bifurcation is observed at $a \simeq 2.355$ associated with a pair of 3-cycles.

In Figure $15 \mathrm{a}$ we see the basin $\mathcal{B}(O)$ together with the basin $\mathcal{B}\left(\mathcal{C}_{3}\right)$, whose frontier is given by the stable set of the 3-cycle saddle. Differently from the previous window, in which we have seen only a coexistent 4-cycle, in this case the 3-cycle will undergo a route to a chaotic attractor, up to its destruction via a contact bifurcation (also called "crisis" in [19]), leaving a "strange repellor" (see also [27]).

In Figure 15b we see the 3-piece cyclical chaotic attractor, whose basin is much wider than $\mathcal{B}(O)$, and we also see that the width of the attracting set $\left\{\mathcal{A}_{1}, \mathcal{A}_{2}, \mathcal{A}_{3}\right\}$ increases with $a$, so that it comes closer and closer to the frontier of its basin, given by $W^{s}\left(S_{i}\right)$ where $\left\{S_{1}, S_{2}, S_{3}\right\}$ is the 3-cycle saddle. In Figure 15c we see how several branches of the chaotic set $\mathcal{A}_{i}$ are approaching $W^{s}\left(S_{i}\right), i=1,2,3$. It is clear that a contact bifurcation will occur, as $a$ is increased, between the chaotic set $\mathcal{A}_{i}$ and $W^{s}\left(S_{i}\right)$, $i=1,2,3$ (see the enlargement in Fig. 15d), causing 


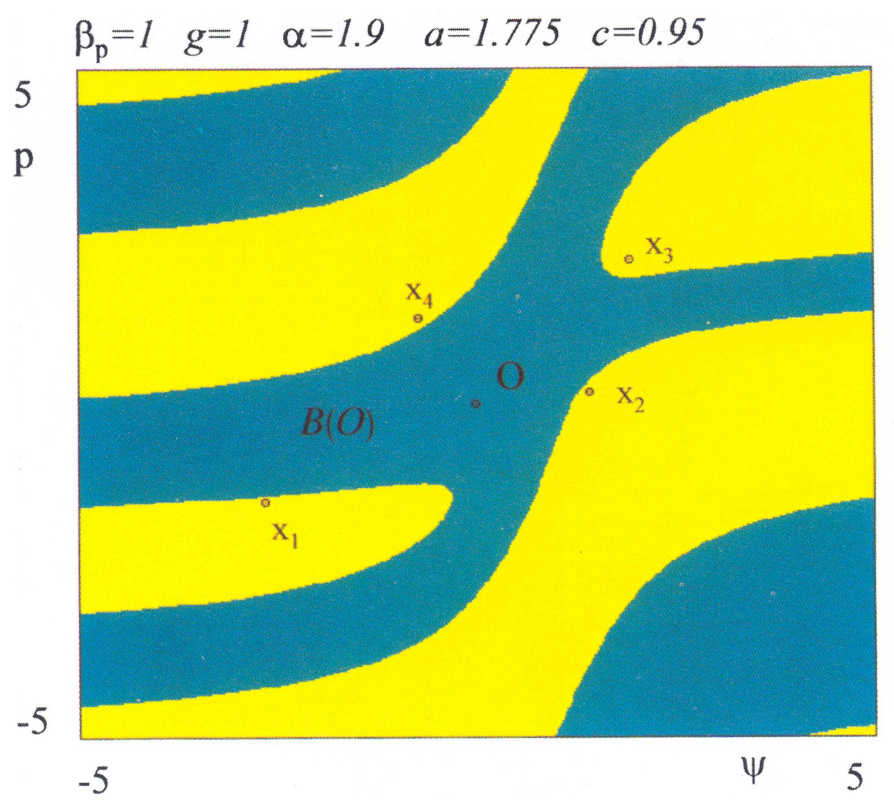

(a)

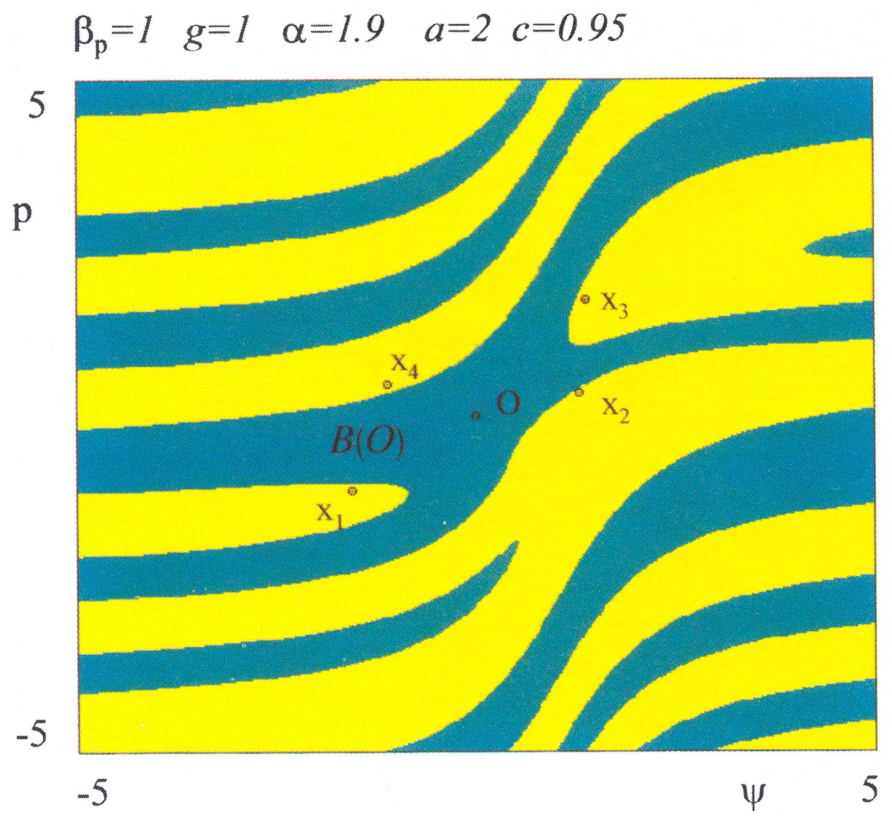

(b)

FIGURE 14 Competition between attracting sets observed for particular combinations of parameters, included in case A of Figure 1. For $c=0.95, \beta_{p}=1, g=1, \alpha=1.9$, and increasing $a$ starting from low values inside the stability region, the origin $O$ is initially the only attracting set, but as $a$ is increased a saddle-node bifurcation is observed. In (a), where $a=1.775$, and in (b), where $a=2$, there is coexistence of the stable fixed point $O$ and a stable 4-cycle $\mathcal{C}_{4}$. (See Color Plate VII.)

the disappearance of the chaotic attractor (after which the origin is again the only attractor), but leaving also a chaotic repellor.
Let us briefly recall the main properties associated with this contact bifurcation, which often is called "crisis" in the literature on dynamical 


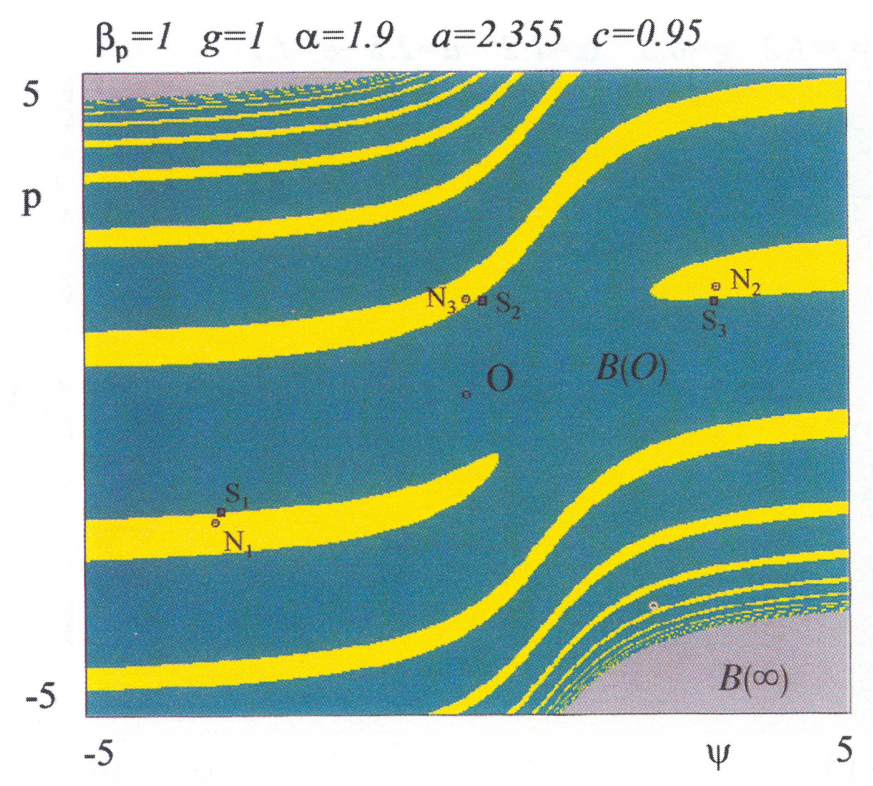

(a)

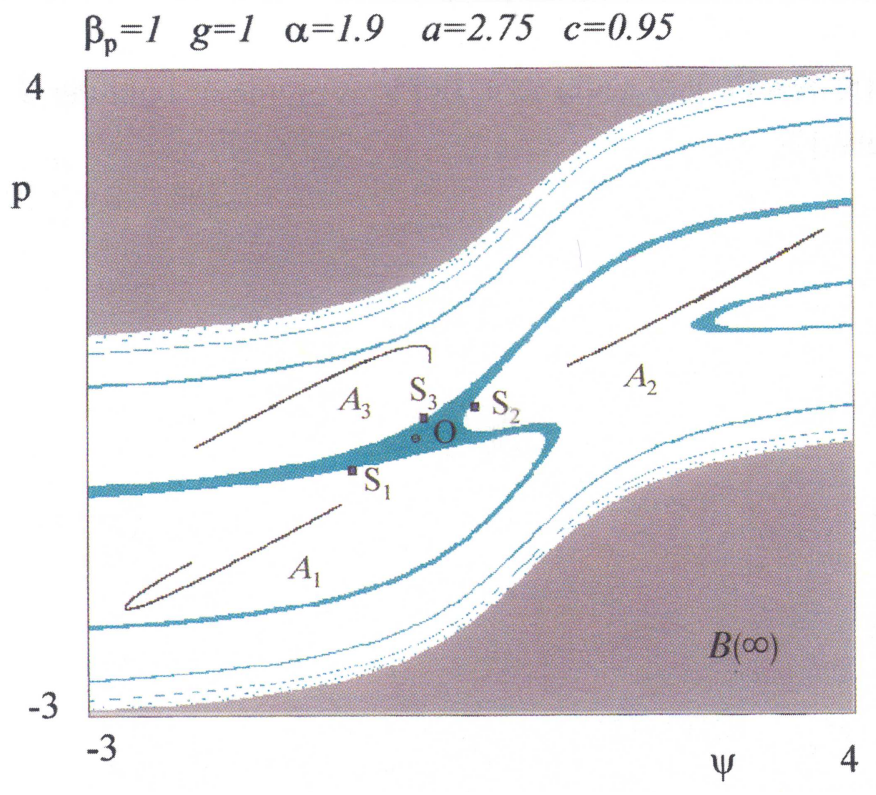

FIGURE 15 The phenomena of persistent coexistence of attracting sets, observed by increasing $a$ further from the situation represented in Figure 14b (where $c=0.95, \beta_{p}=1, g=1, \alpha=1.9$ ). After the disappearance of the 4-cycle represented in that figure, a new competing attracting set appears, a 3-cycle $\mathcal{C}_{3}$ shown in (a), at $a=2.355$, whose basin becomes much wider than $\mathcal{B}(O)$. This 3cycle undergoes a flip-bifurcation sequence, which finally transforms it into a 3-piece chaotic attractor, as shown in (b) for $a=2.75$. This attracting set increases in size, and it approaches the boundary of its basin, as shown in (c), obtained for $a=2.84675$. The enlargement in (d) shows that a "contact bifurcation" or "crisis" is about to occur, destroying the chaotic attracting set, leaving $O$ as the only attractor but leaving also a chaotic repellor in the region of bounded trajectories. The effect of the presence of this chaotic repellor on the transient part of the trajectories is shown in (e), where $a=3.2225$. By increasing $a$ further, the observed sequence of global bifurcations also occurs in a "backward" way: a reverse contact bifurcation transforms again the chaotic repellor into a 3piece chaotic attractor: see (f), obtained for $a=3.225$. It undergoes a sequence of reverse flip-bifurcations, up to the restoration of a 3 -cycle $\mathcal{C}_{3}$ shown in (g), where $a=3.3$. A reverse saddle-node bifurcation occurring at $a \simeq 3.363$ causes the disappearance of the competing 3-cycle, leaving again the fixed point $O$ as unique attracting set, but now with a smaller basin than the one observed for low values of $a$. By increasing $a$ further, a flip bifurcation "replaces" the attracting fixed point with an attracting 2-cycle represented in (h) for $a=3.9196$, whose basin is however reduced to a narrow strip. (See Color Plate VIII.) 

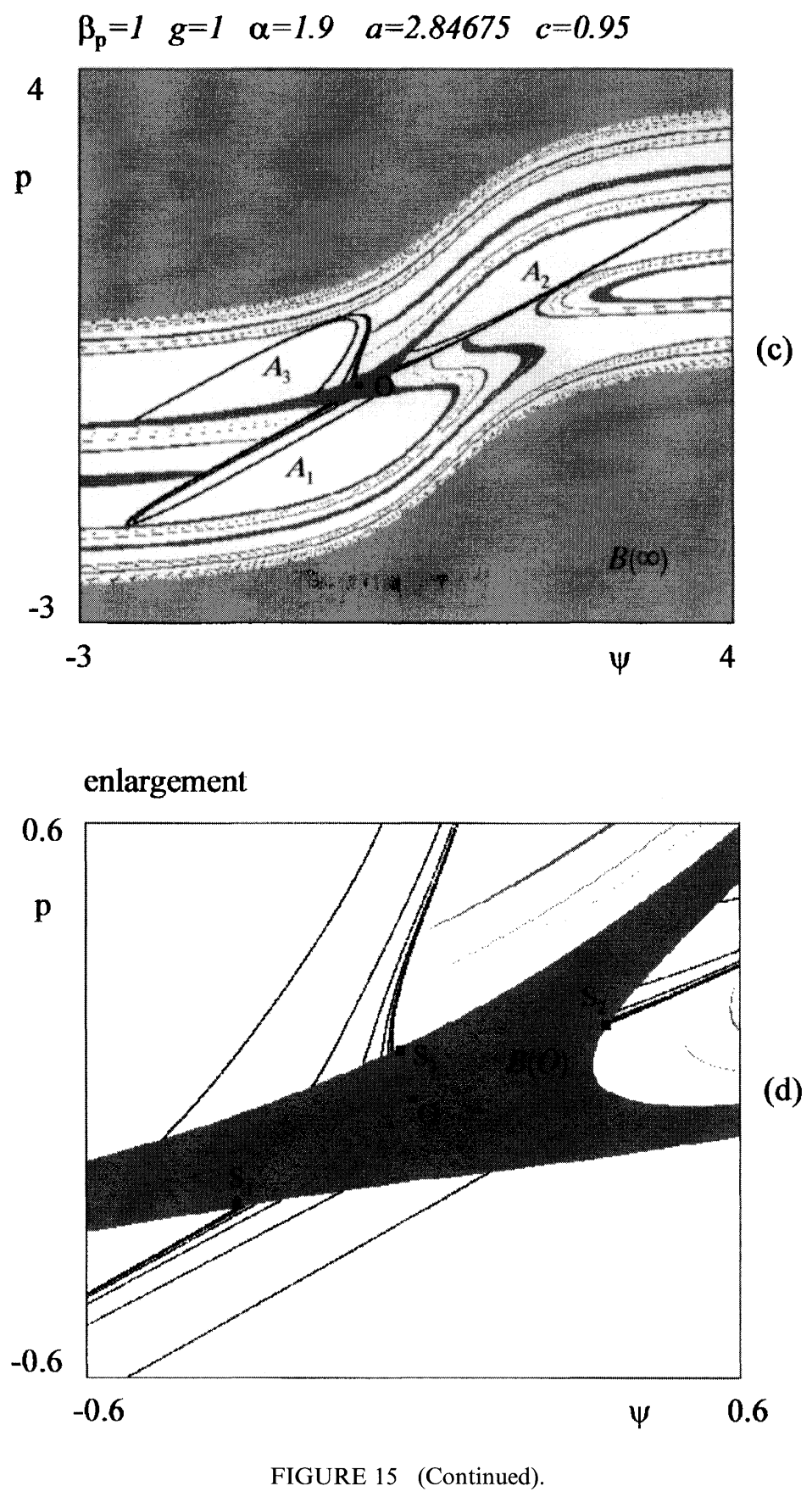

systems, following [19]. It is associated with the homoclinic bifurcation of the saddle 3-cycle on the boundary of the basin. Indeed, the unstable set $W^{u}\left(S_{i}\right), i=1,2,3$, of the saddle cycle is dense on the attractor $\left\{\mathcal{A}_{1}, \mathcal{A}_{2}, \mathcal{A}_{3}\right\}$ and thus a contact between $\mathcal{A}_{i}$ and $W^{s}\left(S_{i}\right)$ also means a tangent bifurcation between $W^{s}\left(S_{i}\right)$ and $W^{u}\left(S_{i}\right), i=1,2,3$, followed by transverse intersection of these 

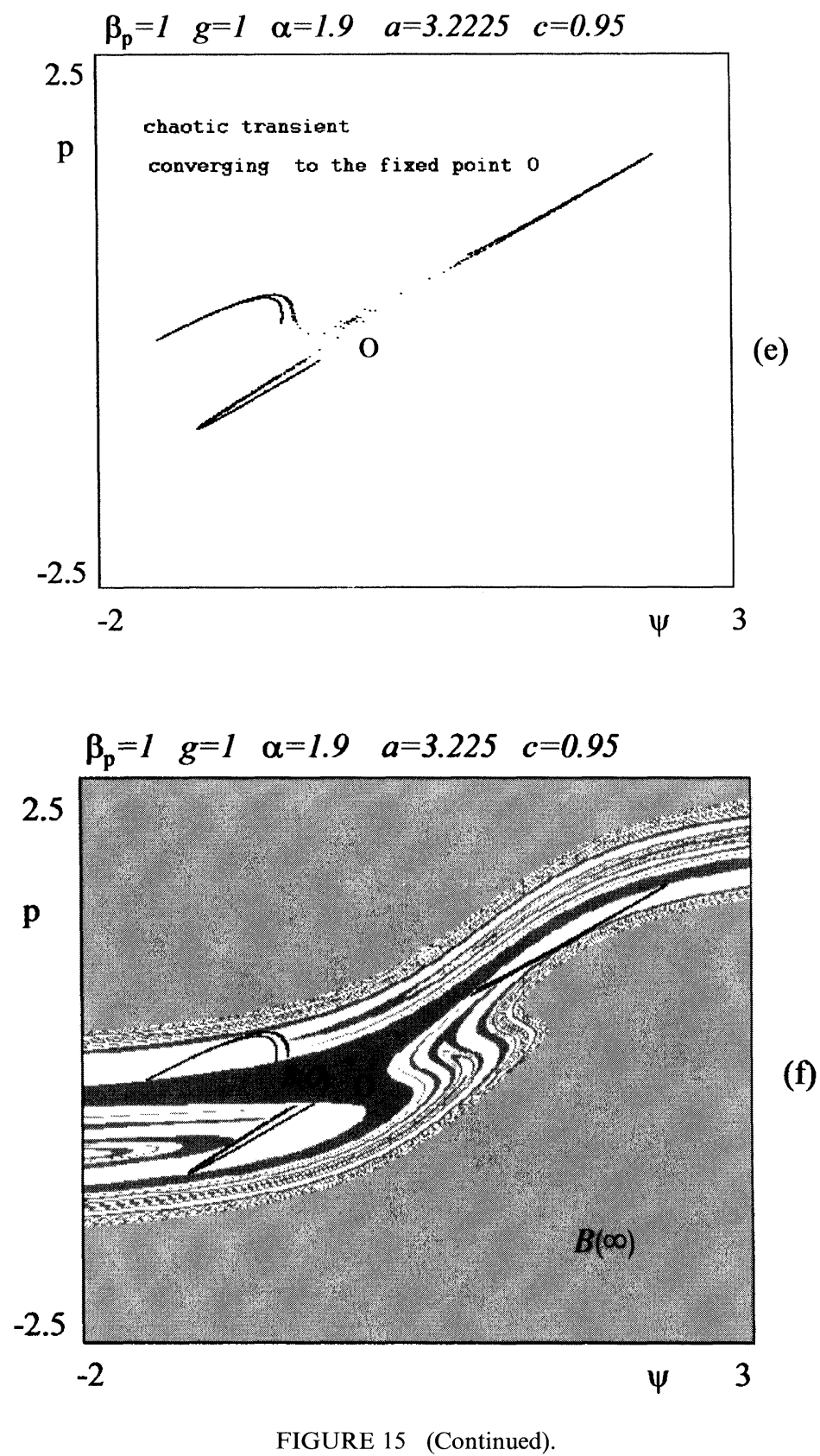

unstable and stable sets. Moreover, we are in a regime of noninvertibility of $T$, and we know, as already remarked in [11] (see also [27]) that such chaotic sets are bounded by images of critical curves, and thus a tangency between $\mathcal{A}_{i}$ and $W^{s}\left(S_{i}\right)$ also denotes a contact between a critical curve $L C_{k}$ and $W^{s}\left(S_{i}\right)$.

The occurrence of this contact indicates that the first homoclinic orbit of a cycle, in noninvertible maps, is often associated with critical homoclinic 


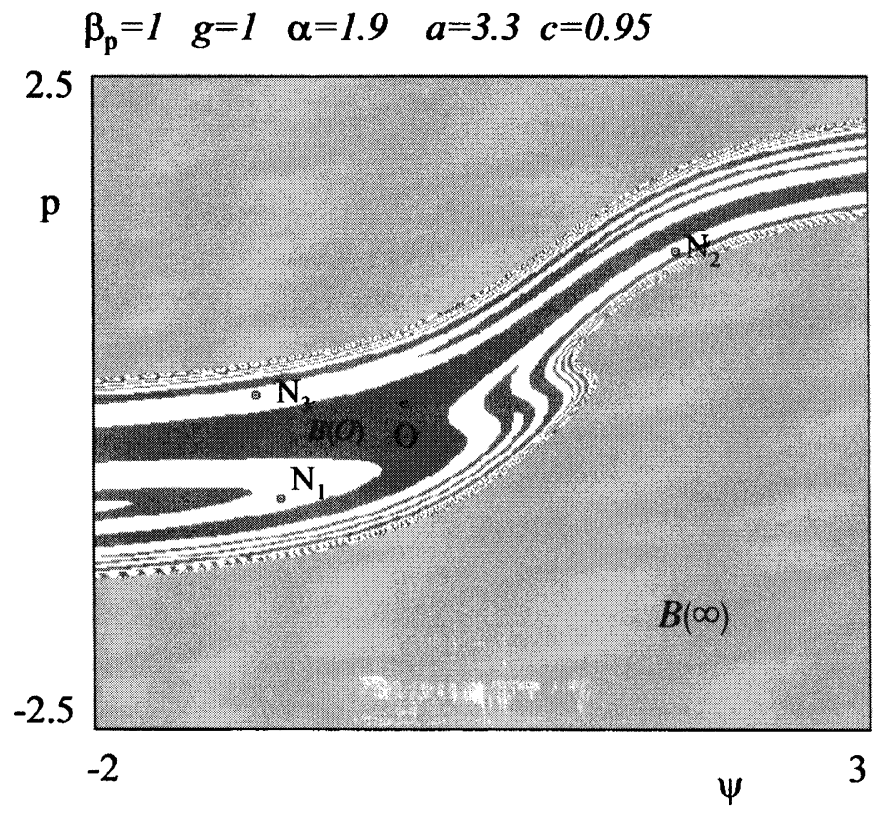

(g)

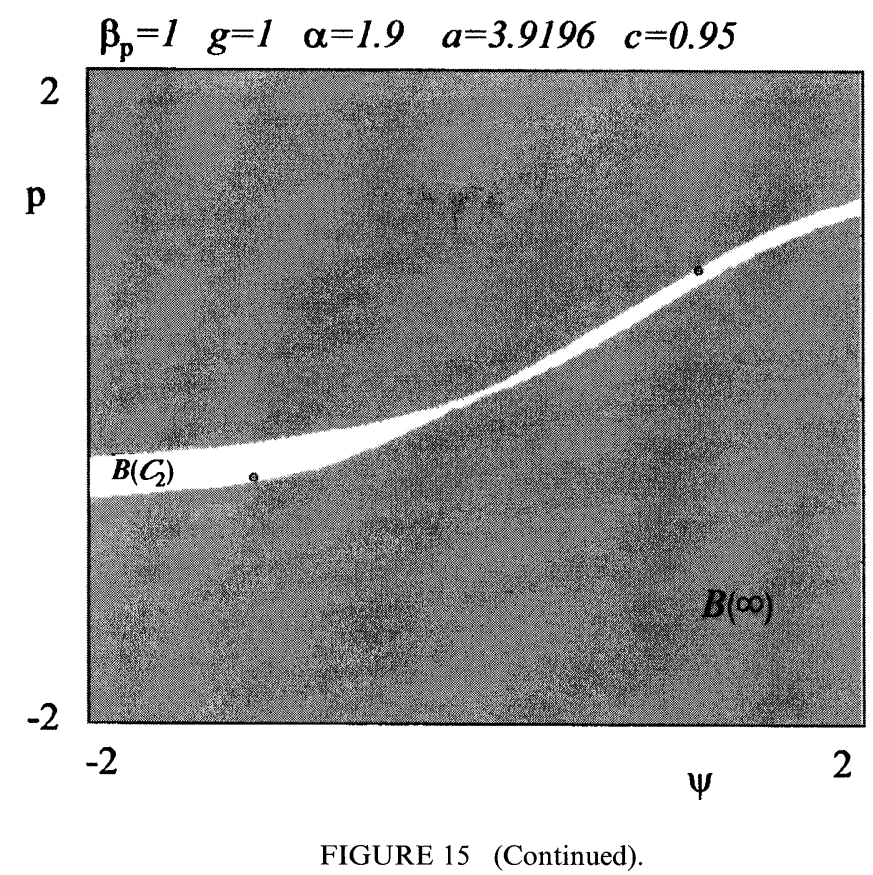

(h)

orbits (i.e., all the homoclinic orbits necessarily have one critical point belonging to $L C_{-1}$ ), as it occurs in one-dimensional maps (see $[13,18])$. It is worth noting the difference between the homoclinic bifurcation of the 3-cycle $\left\{S_{1}, S_{2}, S_{3}\right\}$ on the boundary of the basin, commented in Section 5 , 
with respect to the one just observed. In Section 5 the homoclinic bifurcation only changed the basin boundary without involving the attracting sets (which were the same before and after the bifurcation) while the one described in Figures $15 \mathrm{c}, \mathrm{d}$ also involves the attracting sets.

The destruction of the chaotic attractor leaves a strange repellor in the set of bounded trajectories. This means that the white points in Figure $15 \mathrm{e}$ all converge to the origin except for an "invisible" set of zero Lebesgue measure, on which the restriction of $T$ is chaotic. The existence of such a strange repellor is revealed by the transient part of the generic trajectory, which behaves chaotically before settling down on the origin, as in the example reported in Figure $15 \mathrm{e}$. As the parameter $a$ increases, this forward route to chaos is also observed in the backward way: in Figure 15f we see that a 3-piece chaotic attractor appears again. This means that the homoclinic orbits of the saddle $\left\{S_{1}, S_{2}, S_{3}\right\}$ have disappeared, the intersections between $W^{u}\left(S_{i}\right)$ and $W^{s}\left(S_{i}\right), \quad i=1,2,3$, have caused a backward route leading again, after a homoclinic tangency, to an empty intersection. The backward homoclinic tangency causes the transition of a chaotic repellor into a chaotic attractor (see Fig. 15f).

The three piece chaotic attractor, in its turn, undergoes all the backward bifurcations up to the restoration of an attracting 3-cycle $\left\{N_{1}, N_{2}, N_{3}\right\}$, as shown in Figure $15 \mathrm{~g}$, and up to its backward saddle-node bifurcation causing the merging of the 3-cycle $\left\{N_{1}, N_{2}, N_{3}\right\}$ and the 3-cycle saddle $\left\{S_{1}, S_{2}, S_{3}\right\}$ and the consequent disappearance of these cycles at $a \simeq 3.363$. The fixed point is again the only attractor, but we can see that its basin is now much smaller than the one existing for low values of $a$. Now we are very close to the boundary of the stability region. At $a \simeq 3.719$ the fixed point $O$ undergoes a flip bifurcation giving rise to a stable 2-cycle, but its basin is quite narrow, decreasing more and more as $a$ increases (see Fig. 15h), up to a complete disappearance of attracting sets.

\section{CONCLUSIONS}

We have formulated a discrete time model of a financial market whose dynamics are driven by the interaction of rational fundamentalists and trend chasing chartists, the proportions of these two groups being held fixed. We have analysed the dynamic behaviour of the model by using local stability analysis and performing global inspections of the basins of attraction by means of the theory of critical curves and various numerical tools. We have focussed our attention on the several bifurcations which a basin of attraction, whose size and shape may be considered a kind of "measure" of the "practical stability" of the attracting set, may undergo.

We have also highlighted the crucial trade-offs which affect the dynamics. These being on the one hand the trade off between the strength of fundamentalists' demand $(a)$ and the speed of adjustment of chartists' expectations $(c)$, and on the other hand between the speed of adjustment of market prices $\left(\beta_{p}\right)$ and the strength of chartists' demand at the steady state $\left(k^{\prime}(0)\right)$.

In recent years a number of models of financial markets based on interacting heterogeneous agents have been developed, we cite in particular [7, 22, 8 and 10]. These models are richer and more realistic than ours in that, in one way or another, they allow the size of the different groups of agents to vary according to the evolution of the financial market. These models are therefore of necessity not very mathematically tractable. We believe that our model complements these other studies because it contains the essential elements of the heterogeneous interacting agents paradigm whilst still remaining mathematically tractable. For this reason we have been able to obtain, in Sections 3 6 , a more complete characterization of the global dynamics than is possible in the richer and more realistic models.

Future developments of the model introduced here could focus on attempts of each group to learn about their economic environment in the face of stochastic factors (capturing for example 
the random arrival of new events in the market). Some initial attempts in this direction are outlined in [2], and will be the object of future research.

\section{Acknowledgements}

This work has been performed with the financial support of CNR, Italy, and within the scope of the national research project "Nonlinear Dynamics and Stochastic Models in Economics and Finance", MURST, Italy. Financial support of the Australian Research Council is also acknowledged. The authors wish to thank Anna Agliari and Gian Italo Bischi for useful discussion and comments on the current work. The usual caveat applies.

\section{References}

[1] Abraham, R., Gardini, L. and Mira, C. (1997). Chaos in discrete dynamical systems (a visual introduction in two dimensions). Springer-Verlag, New York.

[2] Agliari, A., Chiarella, C. and Gardini, L. (1999). The feedback between asset prices and learning in a simple model of heterogeneous agents. Mimeo, School of Finance and Economics, University of Technology, Sydney.

[3] Alligood, K. T., Sauer, T. D. and Yorke, J. A. (1997). Chaos, an introduction to dynamical systems. SpringerVerlag, New York.

[4] Bischi, G. I. and Naimzada, A. (1999). Global Analysis of a Duopoly Game with Bounded Rationality. Advances in Dynamic Games and Applications, 5, Birkhauser, $361-385$.

[5] Bischi, G. I., Gardini, L. and Kopel, M. (2000). Analysis of Global Bifurcations in a Market Share Attraction Model. Journal of Economic Dynamics and Control, 24, 855-879.

[6] Brock, W. A. and Hommes, C. H. (1997). A rational route to randomness. Econometrica, 65, 1059-1095.

[7] Brock, W. A. and Hommes, C. H. (1998). Heterogeneous beliefs and routes to chaos in a simple asset pricing model. Journal of Economic Dynamics and Control, 22, $1235-1274$.

[8] Chen, S. H. and Yeh, C. H. (1997). Modelling speculators with genetic programming. In: Evolutionary Programming VI, Lecture Notes in Computer Science, 1213 (Angeline, P. et al., Eds.), Springer-Verlag, New York, 137-147.

[9] Chiarella, C. (1992). The dynamics of speculative behaviour. Annals of Operations Research, 37, 101-123.

[10] Chiarella, C. and He, H. Z. (1999). Heterogeneous beliefs, risk and learning in a simple asset pricing model. Working Paper 18, Quantitative Finance Research Group, School of Finance and Economics, University of Technology, Sydney. Forthcoming in Computational Economics.
[11] Chiarella, C., Dieci, R. and Gardini, L. (2000). Speculative behaviour and complex asset price dynamics. Journal of Economic Behaviour and Organization, to appear.

[12] Chiarella, C., Dieci, R. and Gardini, L. (2000). A dynamic analysis of speculation across two markets. Mimeo, School of Finance and Economics, University of Technology, Sydney.

[13] Devaney, R. L. (1987). An Introduction to Chaotic Dynamical Systems. The Benjamin/Cummings Publishing Co., Menlo Park, California.

[14] De Vilder, R. (2000). On the transition from local regular to global irregular fluctuations. Journal of Economic Dynamics and Control, 24, 247-272.

[15] Fernandez-Rodriguez, F. and Garcia-Artiles, M. D (1998). A model of speculative behaviour with a strange attractor. Mimeo, Departimento de Métodos Cuantitativos para la Economía y Gestión, Universidad de Las Palmas de Gran Canaria.

[16] Frouzakis, C. F., Gardini, L., Kevrekidis, Y. G., Millerioux, G. and Mira, C. (1997). On some properties of invariant sets of two-dimensional non-invertible maps, Int. Journal of Bifurcation and Chaos, 7(6), 1167-1194.

[17] Gandolfo, G. (1996). Economic Dynamics (3rd edn.) Springer-Verlag, New York.

[18] Gardini, L. (1994). Homoclinic bifurcations in $n$ dimensional endomorphisms due to expanding periodic points. Nonlinear Analysis, Theory, Methods and Applications, 23(8), 1039-1089.

[19] Grebogi, C., Ott, E. and Yorke, J. A. (1983). Crises, sudden changes in chaotic attractors and transient chaos. Physica D, pp. 181-200.

[20] Guckenheimer, J. and Holmes, P. (1983). Nonlinear Oscillations, Dynamical Systems, and Bifurcations of Vector Fields. Springer-Verlag, New York.

[21] Gumowski, I. and Mira, C. (1980). Dynamique Chaotique. Cepadues Ed., Toulouse.

[22] Lux, T. (1998). The socio-economic dynamics of speculative markets: interacting agents, chaos and the fat tails of return distributions. Journal of Economic Behaviour and Organization, 33, 143-165.

[23] Li, T. and Yorke, J. A. (1975). Period three implies chaos. American Mathematical Monthly, 82, 985-992.

[24] McDonald, S. W., Grebogi, C., Ott, E. and Yorke, J. A. (1985). Fractal basin boundaries. Physica D, 17, 125-153.

[25] Mira, C., Fournier-Prunaret, D., Gardini, L., Kawakami, H. and Cathala, J. C. (1994). Basin bifurcations of two-dimensional noninvertible maps: fractalization of basins. Int. Journal of Bifurcation and Chaos, 4, 343-381.

[26] Mira, C. and Rauzy, C. (1995). Fractal agregation of basin islands in two-dimensional quadratic noninvertible maps. Int. Journal of Bifurcation and Chaos, 5(4), 991-1019.

[27] Mira, C., Gardini, L., Barugola, A. and Cathala, J. C. (1996). Chaotic Dynamics in Two-Dimensional Noninvertible Maps. World Scientific, Singapore.

[28] Nusse, H. E., Ott, E. and Yorke, J. A. (1995). Saddle-node bifurcations on fractal basin boundaries. Physical Review Letters, 75(13), 2482-2485.

[29] Nusse, H. E. and Yorke, J. A. (1996). Wada basin boundaries and basin cells. Physica D, 90, 242-261.

[30] Nusse, H. E. and Yorke, J. A. (1997). Dynamics: Numerical Explorations. Springer-Verlag, New York.

[31] Puu, T. (2000). Attractors, Bifurcations and Chaos, Springer-Verlag, New York.

[32] Wiggins, S. (1990). Introduction to applied nonlinear dynamical systems and chaos. Springer-Verlag, New York. 


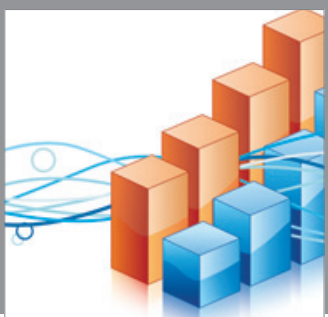

Advances in

Operations Research

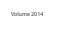

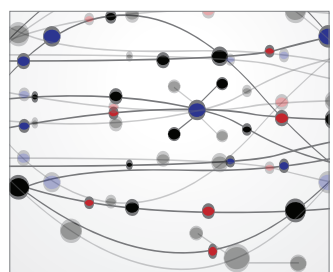

\section{The Scientific} World Journal
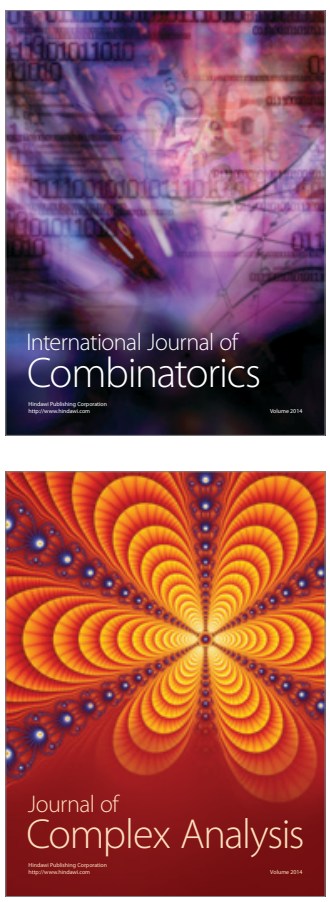

International Journal of

Mathematics and

Mathematical

Sciences
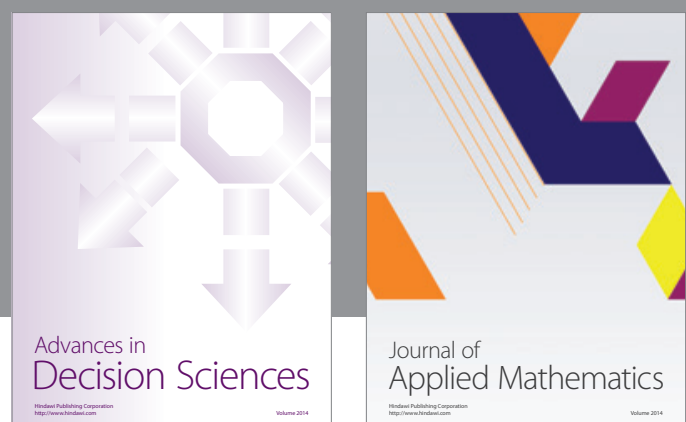

Journal of

Applied Mathematics
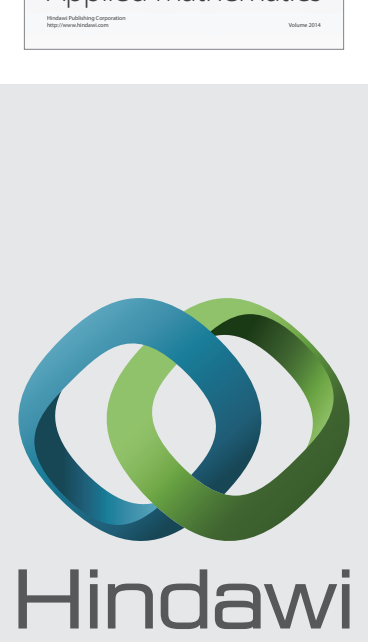

Submit your manuscripts at http://www.hindawi.com
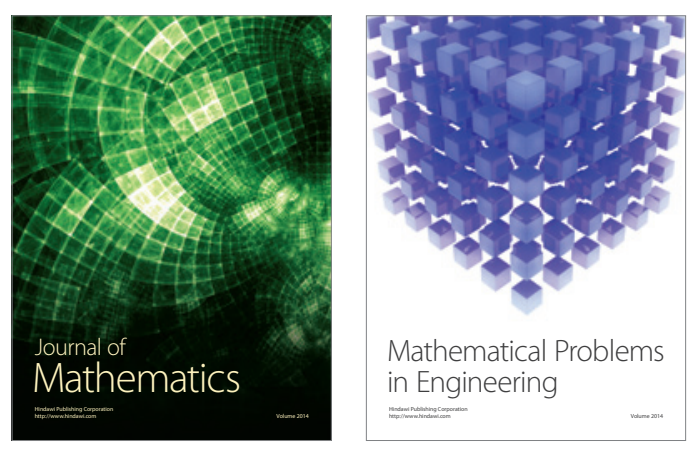

Mathematical Problems in Engineering
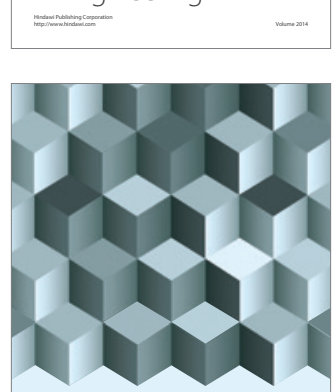

Journal of

Function Spaces
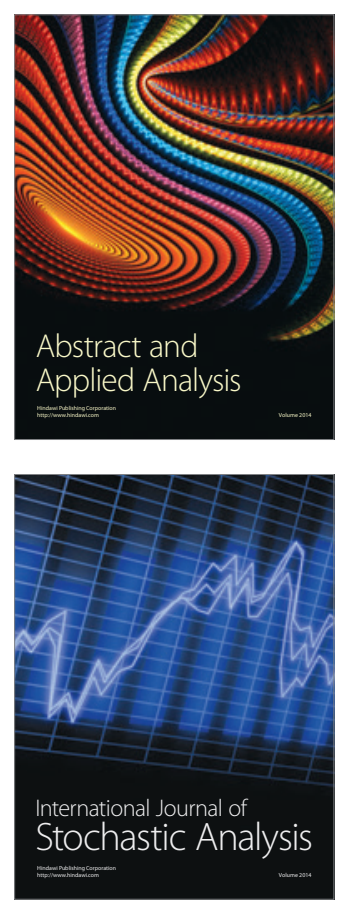

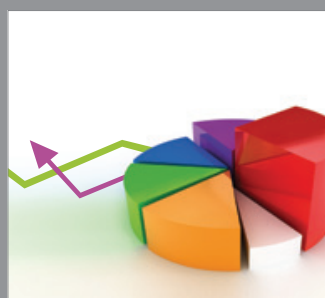

ournal of

Probability and Statistics

Promensencen
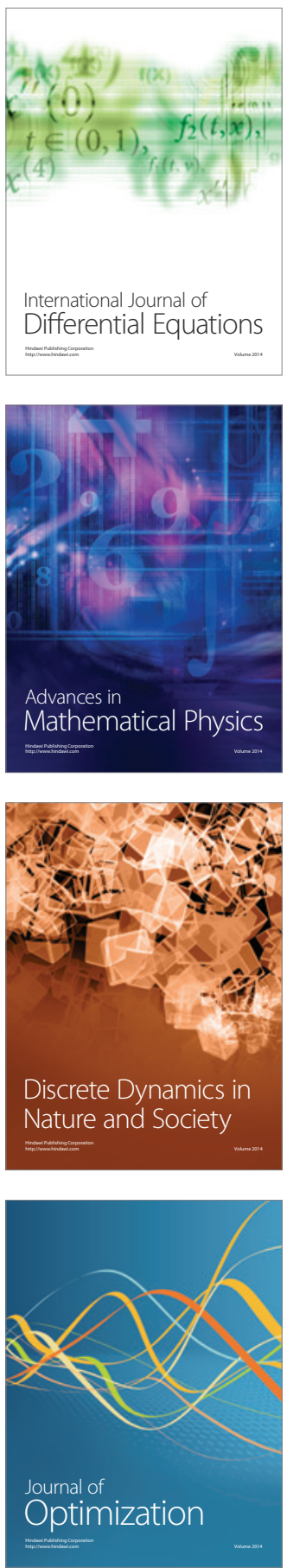